\title{
Depolarization-Dependent C-Raf Signaling Promotes Hyperexcitability and Reduces Opioid Sensitivity of Isolated Nociceptors after Spinal Cord Injury
}

\author{
Anibal Garza Carbajal, Alexis Bavencoffe, ${ }^{\circledR}$ Edgar T. Walters, and ${ }^{\circledR}$ Carmen W. Dessauer \\ Department of Integrative Biology and Pharmacology, McGovern Medical School at University of Texas Health, Houston, Texas 77030
}

Chronic pain caused by spinal cord injury (SCI) is notoriously resistant to treatment, particularly by opioids. After SCI, DRG neurons show hyperactivity and chronic depolarization of resting membrane potential (RMP) that is maintained by cAMP signaling through PKA and EPAC. Importantly, SCI also reduces the negative regulation by Gai of adenylyl cyclase and its production of cAMP, independent of alterations in G protein-coupled receptors and/or G proteins. Opioid reduction of pain depends on coupling of opioid receptors to Gai/o family members. Combining high-content imaging and cluster analysis, we show that in male rats SCI decreases opioid responsiveness in vitro within a specific subset of small-diameter nociceptors that bind isolectin B4. This SCI effect is mimicked in nociceptors from naive animals by a modest 5 min depolarization of RMP $\left(15 \mathrm{mM} \mathrm{K}^{+} ;-45 \mathrm{mV}\right)$, reducing inhibition of cAMP signaling by $\mu$-opioid receptor agonists DAMGO and morphine. Disinhibition and activation of C-Raf by depolarization-dependent phosphorylation are central to these effects. Expression of an activated C-Raf reduces sensitivity of adenylyl cyclase to opioids in nonexcitable HEK293 cells, whereas inhibition of CRaf or treatment with the hyperpolarizing drug retigabine restores opioid responsiveness and blocks spontaneous activity of nociceptors after SCI. Inhibition of ERK downstream of C-Raf also blocks SCI-induced hyperexcitability and depolarization, without direct effects on opioid responsiveness. Thus, depolarization-dependent C-Raf and downstream ERK activity maintain a depolarized RMP and nociceptor hyperactivity after SCI, providing a self-reinforcing mechanism to persistently promote nociceptor hyperexcitability and limit the therapeutic effectiveness of opioids.

Key words: adenylyl cyclase; chronic pain; MAP kinase; nociceptive; opiate; resting membrane potential

Significance Statement

Chronic pain induced by spinal cord injury (SCI) is often permanent and debilitating, and usually refractory to treatment with analgesics, including opioids. SCI-induced pain in a rat model has been shown to depend on persistent hyperactivity in primary nociceptors (injury-detecting sensory neurons), associated with a decrease in the sensitivity of adenylyl cyclase production of cAMP to inhibitory G $\alpha$ i proteins in DRGs. This study shows that SCI and one consequence of SCI (chronic depolarization of resting membrane potential) decrease sensitivity to opioid-mediated inhibition of cAMP and promote hyperactivity of nociceptors by enhancing C-Raf activity. ERK activation downstream of C-Raf is necessary for maintaining ongoing depolarization and hyperactivity, demonstrating an unexpected positive feedback loop to persistently promote pain.

\section{Introduction}

A highly distressing and often permanent complication of traumatic spinal cord injury (SCI) is chronic pain (Widerstrom-

Received Apr. 7, 2020; revised June 16, 2020; accepted July 13, 2020.

Author contributions: A.G.C., E.T.W., and C.W.D. designed research; A.G.C. and A.B. performed research;

A.G.C., A.B., E.T.W., and C.W.D. analyzed data; A.G.C., A.B., E.T.W., and C.W.D. edited the paper; A.G.C., A.B.,

E.T.W., and C.W.D. wrote the paper; C.W.D. wrote the first draft of the paper.

This work was supported by National Institute of Neurological Diseases and Stroke Grant NS091759 to C.W.D. and E.T.W., and Craig H. Neilsen Foundation Grant 545880 to C.W.D. We thank Emily Spence and Max Odem for rat SCl surgeries; and Erica Lee for help with pRKIP analysis.

The authors declare no competing financial interests.

Correspondence should be addressed to Carmen W. Dessauer at Carmen.W.Dessauer@uth.tmc.edu.

https://doi.org/10.1523/JNEUROSCI.0810-20.2020

Copyright $\odot 2020$ the authors
Noga, 2017). Like many forms of chronic pain, SCI-induced pain is often refractory to treatment by available pain medications, including opioids (Bryce, 2018). While alterations within the damaged spinal cord as well as the brain contribute to chronic SCI pain (Kramer et al., 2017; Vierck, 2020), our group and others have shown that electrical activity generated in peripheral terminals and cell bodies of sensory neurons located in DRGs persistently increases after SCI (Carlton et al., 2009; Bedi et al., 2010; Ritter et al., 2015; Odem et al., 2018). Mechanisms of hyperactivity in nociceptors are logical targets for ameliorating chronic SCI pain because Nav1.8 expression (characteristic of most nociceptors and important for their action potential [AP] generation) was found to be necessary for chronic SCI pain in 
rats as measured by reflexive and operant behavioral tests (Yang et al., 2014).

We have previously shown that cAMP signaling is required for maintaining persistent hyperexcitable alterations induced by SCI in nociceptors (Bavencoffe et al., 2016; Berkey et al., 2020). Unexpectedly, the negative regulation of cAMP production at the level of adenylyl cyclase (AC) by G $\alpha$ i proteins was reduced after SCI in membrane preparations from DRGs, independent of alterations in $\mathrm{G}$ protein-coupled receptors and/or $\mathrm{G}$ proteins (Bavencoffe et al., 2016). Analgesic effects of opioids and cannabinoids depend on the coupling of these modulators' receptors to G $\alpha$ i family members. Thus, an SCI-induced decrease in AC sensitivity to $\mathrm{G} \alpha \mathrm{i}$ proteins indicates a reduction in the sensitivity of cAMP production in nociceptors to both endogenous and clinically applied opioids that might exacerbate chronic pain (Sun et al., 2019). How this insensitivity to opioid signaling is produced and how it integrates with the other alterations observed in nociceptor function after SCI are not known.

Our observations that SCI induces chronic depolarization of nociceptors (Bavencoffe et al., 2016; Odem et al., 2018; Berkey et al., 2020) and decreases G $\alpha$ i-mediated inhibition of cAMP production led to the hypothesis that SCI-induced depolarization of resting membrane potential (RMP) plays a major role in driving the reduced responsiveness to opioids. Combining high-content microscopy, cluster analysis, and electrophysiology, we have tested this hypothesis and defined a critical signaling pathway. We confirm that, months after SCI in intact rats, DRG neurons in culture are less sensitive to the $\mu$ opioid receptor (MOR) agonist DAMGO, and we show that this phenomenon is largely restricted to the subpopulation of DRG neurons that bind isolectin B4 (IB4), a marker of nonpeptidergic nociceptors. Importantly, reduced opioid sensitivity is induced in nociceptors from naive rats by artificial depolarization of RMPs similar to the depolarized RMPs found in spontaneously active nociceptors after SCI. The reduced opioid sensitivity is produced at least in part by depolarization activating components of the ERK pathway, including increased phosphorylation of C-Raf (S338) and phosphorylation-induced inhibition of C-Raf inhibitor RKIP (S153). Disinhibition and activation of C-Raf by depolarization shift the dose-response curve of DAMGO to higher concentrations in DRG neurons from naive and SCI rats. Moreover, in nonexcitable HEK293 cells, activation of C-Raf or expression of an activated form of C-Raf also reduces the sensitivity of AC to the effects of opioids. The resulting ERK activity downstream from C-Raf is not necessary for the reduced responsiveness to opioids, but in sensory neurons ERK is required to maintain depolarized RMP and hyperactivity after SCI, demonstrating a positive feedback relationship between depolarization and ERK activity. Our results not only provide a novel mechanism linking opioid insensitivity to nociceptor hyperexcitability in a chronic pain state, but also reveal an unexpected feedback function for the persistent depolarization of RMP that is associated with SCI and many other chronic pain conditions to recurrently stimulate cell signaling pathways important for maintaining the nociceptor depolarization and other hyperexcitable alterations that persistently drive pain.

\section{Materials and Methods}

\section{Antibodies and reagents}

Primary antibodies. Primary antibodies are as follows: rabbit monoclonal anti-phospho RII (S99, 1:1000, clone 151, Abcam, \#ab32390), mouse monoclonal anti phospho-p44/42 mitogen-activated protein kinase (MAPK, T202/Y204, 1:300, clone E10, Cell Signaling Technology, \#9106), anti-phospho RKIP (S153, 1:500, polyclonal, Santa Cruz Biotechnology, \#sc-32 622), anti-phospho C-Raf (S338, 1:500, Cell Signaling Technology, \#9427), anti-CGRP (1:1000, Santa Cruz Biotechnology, \#SC-57 053), anti-PGP9.5 (1:4000, Novus Biologicals, \#NB11058872 ). Secondary antibodies (all 1:1000) goat anti-chicken- DyLight 755, goat anti-mouse AlexaFluor-750, donkey anti-mouse AlexaFluor$647,-568,-488$, and goat anti-rabbit AlexaFluor-568 and -488 were purchased from Thermo Fisher Scientific. IB4-FITC (1:1500, Sigma Millipore, \#L2895) DAPI.

Drugs. Retigabine and inhibitors for C-Raf (GW5074), pan-Raf (RAF709), ERK (UO126), PKA (H89), PKC (sotrastaurin), and Src (saracatinib) were purchased from Selleck Chemicals. Forskolin (Fsk) and morphine sulfate were purchased from Cayman Chemicals. DAMGO was purchased from Bachem. All drugs were prepared as stock solutions in PBS (morphine, DAMGO) or DMSO (retigabine, GW5074, RAF709, UO126, H89, Sotrastaurin, Saracatinib, Fsk) and kept as aliquots at $-20^{\circ}$ C. Raf-CTH was a gift from John Hancock (McGovern Medical School) and represents a fusion of Raf-1 (C-Raf) with the complete carboxy-terminal hypervariable region of $\mathrm{H}$-Ras, resulting in plasma membrane targeting and Raf activation (Inder et al., 2008).

\section{Animals}

All procedures followed the guidelines of the International Association for the Study of Pain and were approved by the McGovern Medical School Animal Care and Use Committees. Male Sprague Dawley rats (Envigo) (8-9 weeks old, 250-300 g, 2 per cage) were allowed to acclimate to a $12 \mathrm{~h}$ reverse light/dark cycle for at least $4 \mathrm{~d}$ before beginning experiments. Sex differences between male and female rats in nociceptor hyperactivity have been noted after SCI (Bedi et al., 2010); and, like other pain-related phenomena (Mogil, 2020), these may involve complex sexspecific mechanisms. Possible sex differences in the mechanisms addressed in this report are under separate investigation by the authors.

\section{SCI procedures}

Surgeries were conducted as previously described (Bedi et al., 2010; Wu et al., 2013; Yang et al., 2014; Bavencoffe et al., 2016; Berkey et al., 2020). Rats were anesthetized with isoflurane (induction 4\%-5\%; maintenance 1\%-2\%, Henry Schein Medical). A T10 vertebral laminectomy was followed by a dorsal contusive spinal impact (150 kilodyne, $1 \mathrm{~s}$ dwell time) using an Infinite Horizon Spinal Cord Impactor (Precision Systems and Instrumentation). Sham-operated rats received the same surgical treatment without the contusion. The analgesic buprenorphine hydrochloride $(0.02 \mathrm{mg} / \mathrm{kg}$ in $0.9 \%$ saline $2 \mathrm{ml} / \mathrm{kg}$; Buprenex, Reckitt Benckiser Healthcare) and the antibiotic enrofloxacin $(0.3 \mathrm{ml}$ in $0.9 \%$ saline; Enroflox, Norbrook) were injected intraperitoneally twice daily for $5 \mathrm{~d}$ (buprenorphine) or $10 \mathrm{~d}$ (enrofloxacin). Manual bladder evacuations were performed twice daily until rats recovered neurogenic bladder voiding. Rats had free access to food and water. All rats included in this study received a score of 0 or 1 for both hind limbs the day after surgery, as measured on the Basso, Beattie, and Bresnahan Locomotor Rating Scale (Basso et al., 1995). Tissue from SCI or Sham rats was harvested 13 months after surgery. In this study, most of the controls were uninjured, naive rats. In our initial experiments, sham-operated rats were also examined and found to show no apparent differences in biochemical responses to opioids compared with sham-operated rats, similar to previous observations of relatively little difference in electrophysiological properties between naive and sham-surgery control groups (Bedi et al., 2010; Bavencoffe et al., 2016; Odem et al., 2018). Naive and sham-surgery controls were therefore pooled into a single Control group for these experiments (see Fig. 1), whereas in the subsequent experiments only a Naive control group was used.

\section{$D R G$ neuron cultures}

DRGs were harvested below vertebral level T10. Ganglia were surgically desheathed before being transferred in high-glucose DMEM culture medium (Sigma Millipore) containing trypsin TRL $(0.3 \mathrm{mg} / \mathrm{ml}$, Worthington Biochemical) and collagenase D $(1.4 \mathrm{mg} / \mathrm{ml}$, Roche Life Science). After $40 \mathrm{~min}$ incubation under constant shaking at $34^{\circ} \mathrm{C}$, digested DRG fragments 
A

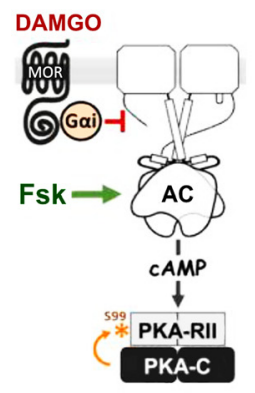

B

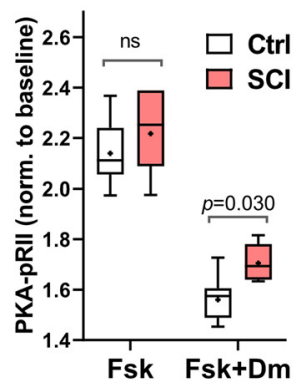

C

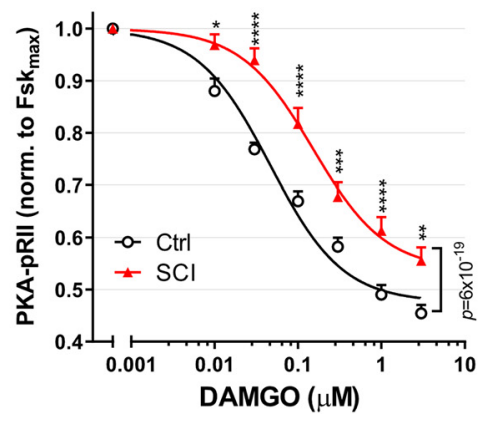

D

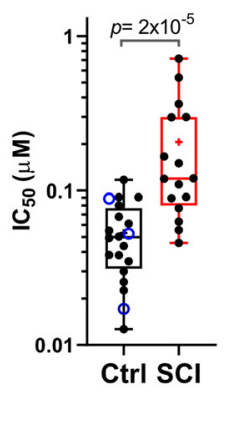

Figure 1. SCI reduces the effect of MOR agonist DAMGO on CAMP signaling. $A$, Phosphorylation of PKA-RIl (S99) is used as a surrogate measurement of cAMP production in response to Fsk activation of AC and inhibition by the G $\alpha$ i/o-coupled MOR agonist, DAMGO. B, DAMGO inhibition of Fsk responses in Control group (Control, white boxes, pooled Naive and Sham groups) and $\mathrm{SCl}$ group (Fsk $3 \mu \mathrm{m}$, DAMGO $0.3 \mu \mathrm{m}, 5 \mathrm{~min}$ ). PKA-pRIl levels are normalized to the baseline of the unstimulated condition in each case. Control, $n=9$; SCl, $n=7$, compared via two-way ANOVA, followed by Sidak's multiple comparisons test. Significant $p$ values are indicated on the graph. Box-and-whisker plot represents the median, mean $(+)$, quartiles, and range of the data. C, Dose-response curves for DAMGO inhibition of Fsk responses (Fsk $3 \mu \mathrm{m}$ ) in Control group (black line) and SCI (red) DRG cultures. PKA-pRII data were normalized to the Fsk response in the absence of DAMG0 after baseline (BL) subtraction $\left[\mathrm{Y}_{\text {Norm }}=(\mathrm{Y}-\mathrm{BL}) /\left(\mathrm{Fsk}_{\mathrm{Max}}-\mathrm{BL}\right)\right]$. $\mathrm{IC}_{50}$ : Control $=0.047 \mu \mathrm{m}(n=17-22) ; \mathrm{SCl}=0.15 \mu \mathrm{m}(n=15-17)$. Difference between groups tested by two-way ANOVA, followed by Sidak's test. ${ }^{*} p<0.05,{ }^{* *} p<0.01,{ }^{* * *} p<0.001,{ }^{* * * *} p<0.0001$. Data are mean $\pm S E M$. $\boldsymbol{D}, I_{50}$ values from individual DAMG0 dose-response curves between Control and SCI cultures; $p=2 \times 10^{-5}$, Mann-Whitney test. Control, $n=20 ; S C I, n=16$. In the control column, Blue empty circles represent IC $C_{50}$ values from Sham controls; black circles represent naive controls. Box-and-whisker plot represents the median, mean $(+)$, quartiles, and range of the data. Detailed statistical information is provided in Table 1.

were washed by two successive centrifugations and triturated with a firepolished glass Pasteur pipette. For imaging experiments, cells were further subjected to BSA gradient centrifugation (15\%) to remove disrupted cell debris. For high-content imaging, cells were plated on 96-well plates (Greiner Bio-One) coated with poly-L-ornithine at a density of $\sim 300-500$ neurons per well and incubated overnight in DMEM $37^{\circ} \mathrm{C}, 5 \% \mathrm{CO}_{2}$ and $95 \%$ humidity in absence of growth factors or supplements. For electrophysiology, cells were plated on $8 \mathrm{~mm}$ glass coverslips coated with poly-L-ornithine (Sigma Millipore) in DMEM without serum or growth factors, and incubated overnight at $37^{\circ} \mathrm{C}, 5 \% \mathrm{CO}_{2}$ and $95 \%$ humidity.

\section{HEK-293 cultures and transfection}

HEK-293 cells stably expressing the MOR were maintained at $37^{\circ} \mathrm{C}$ with $5 \% \mathrm{CO}_{2}$ in DMEM supplemented with $10 \% \mathrm{FBS}$ and $100 \mu \mathrm{g} / \mathrm{ml}$ hygromycin B. Cells were seeded at $2.5 \times 10^{5}$ cells per well in a 6 -well plate and transfected the next day with RAF-CTH or pCDNA3 (10 $\mu$ g total DNA per well) using Lipofectamine 2000 (Invitrogen). Medium was replaced $4 \mathrm{~h}$ after transfection. Cells were replated $24 \mathrm{~h}$ after transfection into 96well plates at $1 \times 10^{4}$ cells per well. Unless indicated, cells were serum starved $4 \mathrm{~h}$ before stimulation. Drug treatments, fixation, and staining were performed $48 \mathrm{~h}$ after transfection as described for neuronal cultures.

\section{Cell treatments and immunofluorescence staining}

Neuronal cultures were treated with the indicated reagents $24 \mathrm{~h}$ after plating. All experiments and incubations were performed at $37^{\circ} \mathrm{C}$. Pharmacological treatments were performed using a modified protocol detailed previously (Isensee et al., 2014). Neurons were pretreated with inhibitors for $30 \mathrm{~min}$ before simultaneous addition of Fsk, extracellular potassium, and/or DAMGO for $5 \mathrm{~min}$. Compounds were added by removing half of the supernatant in the culture well, mixing it with $10 \times$ compound in 96-well V-bottom plates using automatic multichannel pipettes, before addition back to the same culture well to reach final concentrations of compound. Controls were treated similarly, mixing the supernatant with vehicle (PBS \pm DMSO). For compounds dissolved in DMSO, the final DMSO concentration in culture medium was $\leq 0.1 \%$. Cells were fixed with $4 \%$ PFA $\left(10 \mathrm{~min}\right.$ at $\left.22^{\circ} \mathrm{C}\right)$ and washed twice with PBS. Blocking and permeabilization of fixed cells were performed in a single step using blocking buffer ( $1 \%$ BSA, $0.075 \%$ Triton X-100, $1 \mathrm{~h}$, room temperature). Subsequently, the cultures were incubated with primary antibodies diluted in blocking buffer at $4^{\circ} \mathrm{C}$ overnight, washed, and incubated with DAPI (1:100) and secondary Alexa dye-coupled antibodies in blocking buffer $(1: 1000,1 \mathrm{~h}$, room temperature). In the case of IB4 staining (1:1500), incubations were conducted simultaneously or after the secondary antibody staining in IB4 buffer $\left(\mathrm{PBS}+0.1 \mathrm{mM} \mathrm{Ca}^{2+}, 0.1\right.$ $\mathrm{mm} \mathrm{Mg}^{2+}, 0.1 \mathrm{~mm} \mathrm{Mn}{ }^{2+}, 1 \mathrm{~h}$ room temperature) followed by three final PBS washes (10 min, room temperature). After the final wash, the plates were sealed and immediately imaged or stored at $4^{\circ} \mathrm{C}$ until imaging.

\section{Quantitative high-content microscopy}

We used a modification of the protocols implemented by Isensee et al. (2014, 2017, 2018). Stained cultures in 96-well plates were scanned using a Cellomics CX5 microscope (Thermo Fisher Scientific). Images of $1104 \times 1104$ pixels were acquired with a $10 \times$ objective and analyzed using the Cellomics software package (Thermo Fisher Scientific). After background correction, neurons were identified based on PGP 9.5 staining intensity. Object segmentation was performed using the geometric method. When required, spillover between channels was compensated in the postanalysis using raw fluorescence data from fluorescence controls (PGP 9.5 alone; PGP 9.5 + antibody 1; and PGP 9.5 + antibody 2). The slope of best linear fit was determined by linear regression (Prism, GraphPad) and used to compensate spillover as described previously (Roederer, 2002). Results for each condition consist of at least three different replicate experiments performed on different days.

\section{Electrophysiology}

Whole-cell patch clamp recordings were performed at $\sim 21^{\circ} \mathrm{C} 18-30 \mathrm{~h}$ after dissociation using an EPC10 USB (HEKA Elektronik) amplifier. Patch pipettes were made of borosilicate glass capillaries (Sutter Instrument) with a horizontal P-97 puller (Sutter Instrument) and firepolished with a MF-830 microforge (Narishige) to a final pipette resistance of 3-8 $\mathrm{M} \Omega$ when filled with an intracellular solution composed of the following (in mM): $134 \mathrm{KCl}, 1.6 \mathrm{MgCl}_{2}, 13.2 \mathrm{NaCl}, 3 \mathrm{EGTA}, 9$ HEPES, $4 \mathrm{Mg}$-ATP, and $0.3 \mathrm{Na}-\mathrm{GTP}$, adjusted to $\mathrm{pH} 7.2$ with $\mathrm{KOH}$ and $300 \mathrm{mOsM}$ with sucrose. Isolated neurons with a soma diameter $\leq 30$ $\mu \mathrm{M}$ were observed at $20 \times$ magnification on IX-71 (Olympus) inverted microscope and recorded in a bath solution containing the following (in $\mathrm{mm}$ ): $140 \mathrm{NaCl}, 3 \mathrm{KCl}, 1.8 \mathrm{CaCl}_{2}, 2 \mathrm{MgCl}_{2}, 10$ HEPES, and 10 glucose, which was adjusted to $\mathrm{pH} 7.4$ with $\mathrm{NaOH}$ and $320 \mathrm{mOsM}$ with sucrose. After obtaining a tight seal $(>3 \mathrm{G} \Omega)$, the plasma membrane was ruptured to achieve whole-cell configuration under voltage clamp at $-60 \mathrm{mV}$. All subsequent recording was under current clamp using Patchmaster version 2x90.1 (HEKA Elektronik). Depolarizing spontaneous fluctuations (DSFs) of membrane potential were measured with a custom-automated program (SFA_pub.py). Procedures for measurements of DSFs (minimum cutoffs, $1.5 \mathrm{mV}$ amplitude, $10 \mathrm{~ms}$ duration), spontaneous activity (SA) at RMP, ongoing activity (OA) at a holding potential of $-45 \mathrm{mV}$, AP voltage threshold, and rheobase at a holding potential of 
$-60 \mathrm{mV}$ were described by Odem et al. (2018). To permit direct comparison with our previous publications and many others, the liquid junction potential (calculated to be $\sim 4.3 \mathrm{mV}$ using pClamp software and the algorithm developed by Barry, 1994) was not corrected. This means that actual membrane potentials were $\sim 4 \mathrm{mV}$ more negative than the values reported in this article.

\section{Data analysis}

One- and two-dimensional probability density plots were generated using FlowJo (Becton Dickinson). Gating of subpopulations was performed by setting thresholds at local minima of probability density plots. Individual cells used to perform the cluster analysis were normalized between the minimal $(0.001 \%)$ and maximal $(0.999 \%)$ fluorescence levels per channel, per experiment. Cluster analysis ( $k$-medians) was performed using software Cluster 3.0 (de Hoon et al., 2004). Three-dimensional (3D) plots were constructed using Plotly (Chart Studio). Data analysis and graph plots were performed using Prism (version 7.03 and 8.0, GraphPad Software).

\section{Statistics}

High Content Microscopy. Data averaged across all neurons of a given type from a single animal represent a single data point $(n=1)$. For cultured cell lines, every plate from independent cultures is considered $(n=1)$. Repetition of the same treatment within an experiment from a single animal or plate provides internal replicates, and the average is reported with $n=1$. At least three independent experiments from different animals, performed on separate dates $(n=3)$, are reported for every dataset. High content microscopy data are presented as mean \pm SEM or box-and-whisker plots indicating the full data range, quartiles, median, and mean. All datasets were tested for normality with the Shapiro-Wilk test. Outliers to be excluded from analysis were identified using the ESD method $(p<0.05)$. Normally distributed data were tested with parametric tests: $t$ test, one-way or two-way ANOVA followed by Tukey's test, Dunnett's multiple comparisons test, or Sidak's multiple comparisons test for each pairwise comparison. Statistical significance was set at $p<0.05$, and all reported values are two-tailed. In the case of non-normally distributed data (see Fig. 1D), data were compared using the Mann-Whitney test. Statistical analysis and fits of dose-response curves were performed using Prism version 8.01 (GraphPad Software).

Electrophysiology. All data are presented as mean \pm SEM, except where noted for electrophysiological measurements of OA (presented as incidence [\%] of neurons sampled). All datasets were tested for normality with the Shapiro-Wilk test. Normally distributed data were tested with parametric tests one-way ANOVA or Brown-Forsythe and Welch ANOVA tests followed, respectively, by Holm-Sidak's or Dunnett's multiple comparisons tests for each pairwise comparison using Prism version 8 (GraphPad Software). Non-normally distributed sets of data were tested with Kruskal-Wallis test followed by Dunn's multiple comparison test. Comparisons of incidence were made using Fisher's exact test with Bonferroni corrections for multiple comparisons. Statistical significance was set at $p<0.05$, and all reported values are two-tailed. Details of all statistical analysis can be found in Table 1 .

\section{Results}

\section{SCI reduces the opioid sensitivity of $\mathrm{IB}^{+}$nociceptors}

Previous biochemical data show a decreased sensitivity to G $\alpha$ imediated inhibition of AC activity in DRG membranes after SCI (Bavencoffe et al., 2016). The reduced sensitivity was receptor-independent, as AC activity was tested with exogenously added purified GTP $\gamma \mathrm{S}$-activated G proteins. To test whether this extends to regulation by opioids of the intact cell bodies of DRG neurons, DRG neurons were cultured overnight after isolation from SCI and control rats 1-3 months after injury. Production of cAMP was induced by Fsk ( $3 \mu \mathrm{M}, 5 \mathrm{~min})$, while opioid responses were simultaneously stimulated by the highly selective MOR agonist DAMGO. Using phosphorylation of the RII regulatory subunit of PKA (PKA-pRII; Fig. 1A) as a surrogate measure of cAMP responses (for details, see Isensee et al., 2018), DAMGO effects were calculated as the inhibition of Fsk-induced phosphorylation of PKA-RII. Extending our previous observations on isolated DRG membranes using activated G $\alpha$ i proteins (Bavencoffe et al., 2016), DAMGO effects on cAMP production were reduced in cultured neurons from SCI rats (Fig. $1 B ; p<0.05$ ), yielding a 3 to 6-fold increase in the $\mathrm{IC}_{50}$ for DAMGO in the SCI group compared with the pooled (Naive and Sham-operated) Control group (Fig. $1 C, D ; \quad \mathrm{IC}_{50}, \quad$ Control $=0.053 \pm 0.0063$, SCI = $0.21 \pm 0.047$, Mann-Whitney test, $p=2 \times 10^{-5}$ ). No significant differences were found between the Naive and Sham groups (not shown).

Because DRG neurons constitute a highly heterogeneous population with discrete neuronal types associated with specific markers and functional specializations, we proceeded to analyze SCI versus Naive responses to DAMGO in distinct neuronal subgroups in culture. To this end, we used a combination of high content microscopy data and $k$-means cluster analysis to separate DRG neurons into different subpopulations based on size (cell body area), CGRP expression (characteristic of peptidergic nociceptors), and IB4 binding (a marker of nonpeptidergic nociceptors). These three parameters were combined in a $3 \mathrm{D}$ space plot where every neuron was represented by coordinates for its area, CGRP expression, and degree of IB4 binding (Fig. 2A). The phosphorylation of PKA-pRII was then mapped onto these 3D coordinates to reveal differences in cAMP signaling for each cluster (Fig. 2B). DRG neurons cultured from both naive and SCI rats could be differentiated into 7 distinct clusters (Fig. $2 A$, $C)$, with no significant differences in cluster sizes between SCI and Naive groups (Fig. 2D). As expected, cAMP/PKA-pRII responses to Fsk were observed in all 7 neuronal clusters, with particular prominence in the IB $4^{+} / \mathrm{CGRP}^{-}$subgroups (Fig. $2 E$ ). Significant DAMGO inhibition of Fsk responses was present in medium-sized and small neurons in $\mathrm{IB}^{+}{ }^{+}$and $\mathrm{CGRP}^{+}$neurons from naive animals, confirming previous reports of MOR mRNA expression in both small $\mathrm{IB}^{+}$and IB4 ${ }^{-}$DRG neurons (Wang et al., 2010). While no significant differences in maximal Fsk responses were observed between SCI and Naive groups, DAMGO effects were specifically impaired in $\mathrm{IB}^{+} / \mathrm{CGRP}^{-}$neurons (IB4) from SCI animals (Fig. $2 E$ ), with a $\sim 50 \%$ reduction in the overall DAMGO effect compared with $\mathrm{IB} 4^{+}$neurons from naive animals ( $54 \%$ vs $27 \%$ inhibition, $p=0.014$, unpaired $t$ test). Within the $\mathrm{IB}^{+} / \mathrm{CGRP}^{+}$group (IB-CG), we also observed a trend toward reduced DAMGO responses after SCI $(p=0.053$, unpaired $t$ test).

\section{Acute depolarization reduces the opioid sensitivity of $\mathrm{IB}^{+}$ nociceptors}

We have previously shown that capsaicin-sensitive and IB4 ${ }^{+}$ DRG neurons (probable nociceptors) dissociated from SCI animals show multiple hyperexcitable alterations, including depolarized RMP, reduced thresholds for generating APs, and an increased occurrence of SA, which normally is quite rare in primary nociceptors (Bedi et al., 2010; Odem et al., 2018). Electrical activity in nociceptors can be promoted by the activation of multiple downstream signaling cascades (Salzer et al., 2019). An interesting general question is whether some of these cascades can also be activated recurrently by the ongoing depolarization of RMP that usually occurs in hyperactive nociceptors (Bavencoffe et al., 2016; Odem et al., 2018; Berkey et al., 2020). Specifically, we asked whether 5 min depolarization of RMP in nociceptors isolated from naive rats, produced by increasing extracellular $\mathrm{K}^{+}$concentration $\left(\left[\mathrm{K}^{+}\right]_{\mathrm{e}}\right)$, is sufficient to decrease 
Table 1. Statistical analysis per figure

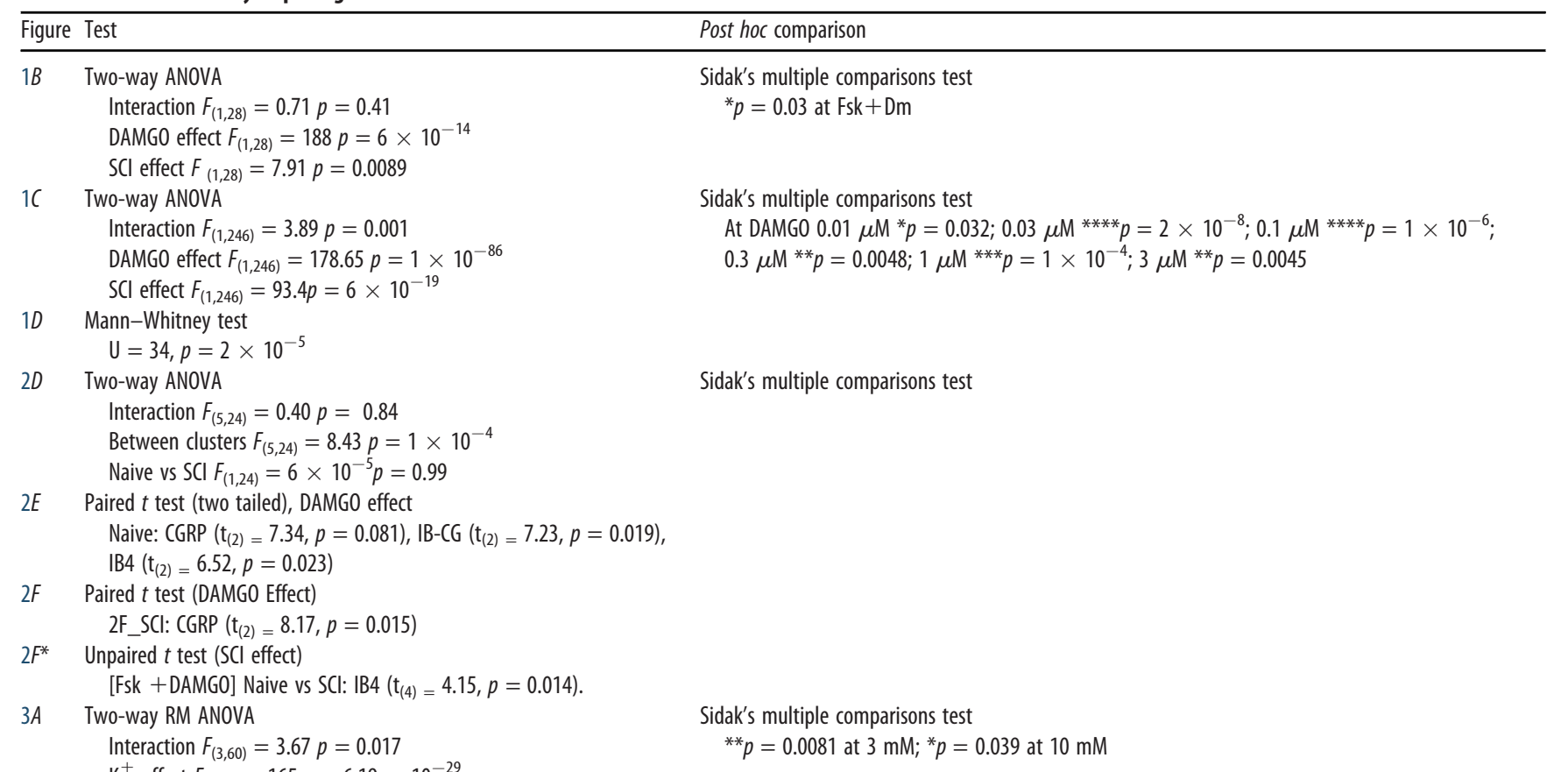

$$
{ }^{* *} p=0.0081 \text { at } 3 \mathrm{mM} ;{ }^{*} p=0.039 \text { at } 10 \mathrm{mM}
$$

$\mathrm{K}^{+}$effect $F_{(3,60)}=165 p=6.19 \times 10^{-29}$

$\mathrm{SCl}$ effect $F_{(1,20)}=5.4 p=0.031$

$3 B$ Two-way ANOVA

Interaction $F_{(4,46)}=1.14 p=0.35$

$\mathrm{K}^{+}$effect $F_{(4,46)}=53.51 p=1 \times 10^{-17}$

SCl effect $F_{(1,46)}=10.51 p=0.0022$

$3 C$ Two-way ANOVA

Interaction $F_{(4,46)}=0.079 p=0.99$

$\mathrm{K}^{+}$effect $F_{(4,46)}=6.4 p=4 \times 10^{-4}$

$\mathrm{SCl}$ effect $F_{(1,46)}=0.25 p=0.62$

3D Two-way ANOVA

Interaction $F_{(1,8)}=4.87 p=0.058$

IB4 effect $F_{(1,8)}=19.55 p=0.0022$

$\mathrm{SCl}$ effect $F_{(1,8)}=3 p=0.12$

$3 E$ Two-way ANOVA

Interaction $F_{(2,30)}=3.7 p=0.036$

Stimuli effect $F_{(2,30)}=120.2 p=5 \times 10^{-15}$

$\mathrm{K}^{+}$effect $F_{(1,30)}=14.63 p=6 \times 10^{-4}$

$3 F$ Two-way ANOVA

Interaction $F_{(6,158)}=13.35 p=3 \times 10^{-12}$

DAMG0 effect $F_{(6,158)}=90.18 p=2 \times 10^{-48}$

$\mathrm{K}^{+}$effect $F_{(1,158)}=211.8 p=6 \times 10^{-31}$

$3 G$ Two-way ANOVA

Interaction $F_{(6,49)}=13.35 p=0.55$

DAMGO effect $F_{(6,49)}=90.18 p=1 \times 10^{-11}$

$\mathrm{K}^{+}$effect $F_{(1,49)}=211.8 p=9 \times 10^{-4}$

$4 A$ One-way ANOVA

$F_{(7,47)}=7.54, p=4 \times 10^{-6}$

$4 A^{\#} \quad$ One-way ANOVA

$F_{(6,37)}=7.54, p=8 \times 10^{-4}$

$4 B$ Two-way ANOVA

Interaction $F_{(15,266)}=11.2 p=5 \times 10^{-21}$

DAMGO effect $F_{(5,266)}=191.4 p=6 \times 10^{-86}$

Treatment effect $F_{(3,266)}=133.3 p=1 \times 10^{-52}$

$4 B \quad$ Two-way ANOVA

Interaction $F_{(10,229)}=13.25 p=3 \times 10^{-18}$

DAMGO effect $F(5,229)=118.64 p=2 \times 10^{-61}$

Treatment effect $F_{(2,229)}=163.3 p=9 \times 10^{-45}$
Sidak's multiple comparisons test

Sidak's multiple comparisons test

Sidak's multiple comparisons test (Naive vs SCI)

$\mathrm{IB} 4{ }^{+}:{ }^{*} p=0.047$

Sidak's multiple comparisons test

[Control]: $p=0.041$; [Fsk+DAMG0]: $p=0.0015$

Sidak's multiple comparisons test

At DAMGO $0.03 \mu \mathrm{M}{ }^{* * * *} p=4 \times 10^{-5} ; 0.1 \mu \mathrm{M}{ }^{* * * *} p=4 \times 10^{-8} ; 0.3 \mu \mathrm{M}{ }^{* * *} p=1 \times$ $10^{-12} ; 1 \mu \mathrm{M}^{* * * *} p=2 \times 10^{-15} ; 3 \mu \mathrm{M}^{* * * *} p<0.0001$

Sidak's multiple comparisons test

Sidak's multiple comparisons test (vs [Control] group)

$[-]:{ }^{* * *} p=5 \times 10^{-4},\left[\mathrm{Src}_{\mathrm{i}}\right]{ }^{*} p=0.014,\left[\mathrm{PKC}_{\mathrm{i}}\right]{ }^{*} p=0.015,\left[\mathrm{PKA}_{\mathrm{i}}\right]^{* * * *} p=7 \times 10^{-6},\left[\mathrm{MEK}_{\mathrm{i}}\right]$ ${ }^{* * *} p=2 \times 10^{-4}$

Dunnett's multiple comparisons test (vs [-] group)

C-Rafi ${ }^{\# \#} p=0.0082$

Tukey's multiple comparisons test.

[Control] vs $\left[\mathrm{K}^{+} 15\right]: p=1 \times 10^{-13},\left[\right.$ Control] vs $\left[\mathrm{K}^{+} 15+\mathrm{GW} 5074\right]: p=2 \times 10^{-3},\left[\mathrm{~K}^{+} 15\right]$ vs $\left[\mathrm{K}^{+} 15+\mathrm{GW} 5074\right]: p=1 \times 10^{-4}$

Sidak's multiple comparisons test (Control + GW5074 excluded)

[Control] vs $\left[\mathrm{K}^{+} 15\right]: 0.03 \mu \mathrm{M} p=3 \times 10^{-5} ; 0.1 \mu \mathrm{M} p=1 \times 10^{-12} ; 0.3 \mu \mathrm{M} p=4 \times 10^{-15}$; $1 \mu \mathrm{M} p<0.0001 ; 3 \mu \mathrm{M} p<0.0001$

$\left[\mathrm{K}^{+} 15\right]$ vs $\left[\mathrm{K}^{+} 15+\mathrm{GW} 5074\right]: 0.1 \mu \mathrm{M} p=5 \times 10^{-5}$.

[Control] vs $\left[\mathrm{K}^{+} 15+\mathrm{GW} 5074\right]: 0.3 \mu \mathrm{M} p=0.0015 ; 1 \mu \mathrm{M} p=6 \times 10^{-15} ; 3 \mu \mathrm{M} p=7 \times 10^{-8}$ 
Table 1. Continued

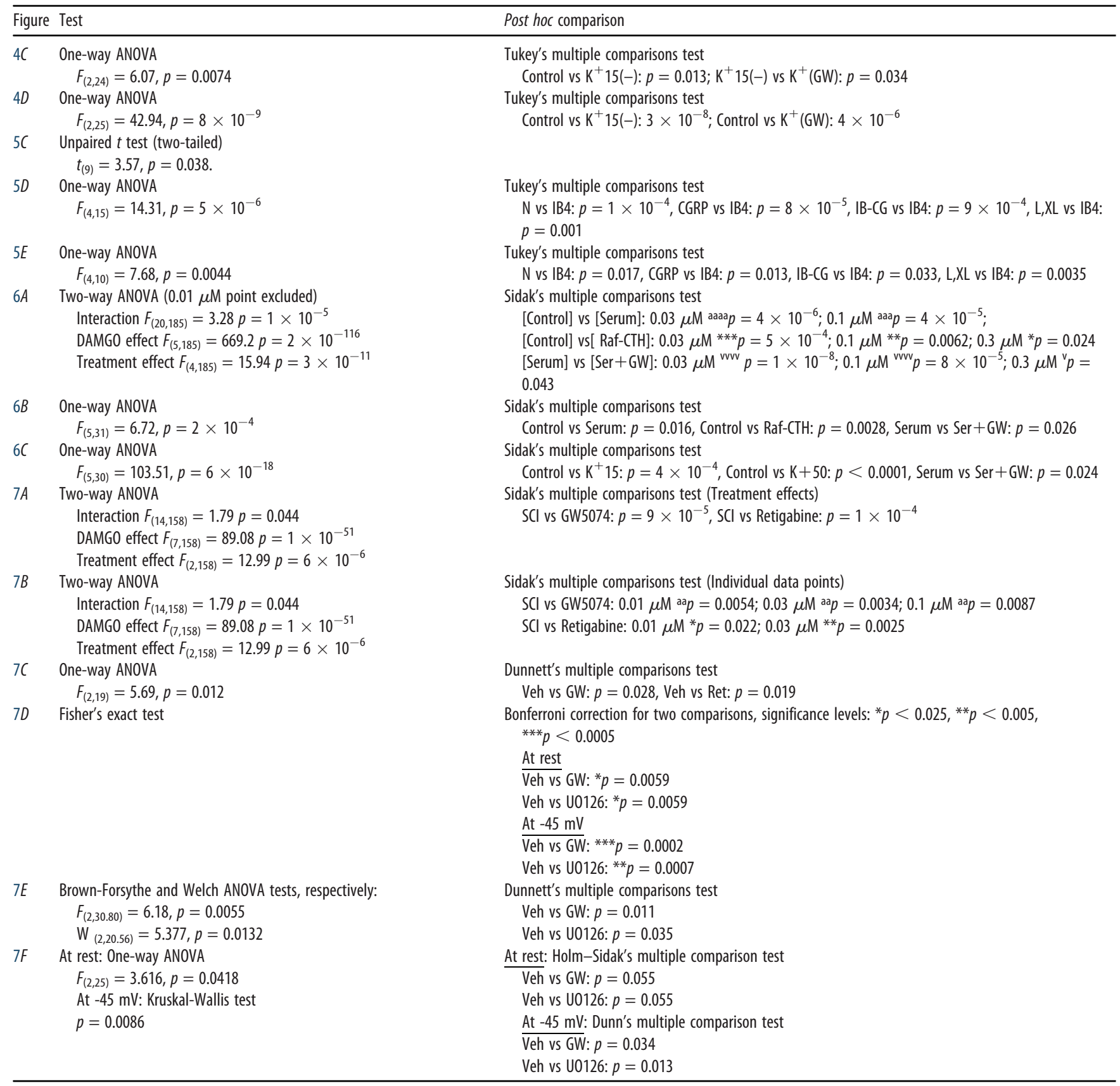

neuronal sensitivity to DAMGO. RMP measured at several $\left[\mathrm{K}^{+}\right]_{\mathrm{e}}$ (Fig. $3 A$ ) was associated with changes in ERK and PKA responses in the general DRG neuronal population (Fig. $3 B, C$ ). Interestingly, neurons in the SCI group exhibited a significantly more depolarized RMP compared with the Naive group at normal (3 mM) and modestly elevated (10 mM) extracellular $\mathrm{K}^{+}$concentrations (Fig. 3A), but the RMPs converged at higher $\left[\mathrm{K}^{+}\right]_{\mathrm{e}}$. In agreement with previous findings in PC12 cells and primary cortical neurons (Rosen et al., 1994), increasing $\left[\mathrm{K}^{+}\right]_{\mathrm{e}}$ activated ERK by phosphorylation in both Naive and SCI groups. Significant ERK activation was found at RMPs at or more depolarized than $-45 \mathrm{mV}\left(15 \mathrm{~mm}\left[\mathrm{~K}^{+}\right]_{\mathrm{e}}\right)$, suggesting a threshold for ERK activation near $-45 \mathrm{mV}$ in the Naive and SCI groups, with an additional enhancement of ERK activation by SCI above this threshold (Fig. 3B; $p=0.0022$, two-way ANOVA). The depolarization-enhanced phosphorylation of ERK occurred at much lower $\left[\mathrm{K}^{+}\right]_{\mathrm{e}}(13-15$ $\mathrm{mM}$, resulting in RMPs of $\sim-50$ to $-40 \mathrm{mV}$ ) than those used in studies of other cell types (e.g., $50-100 \mathrm{~mm}\left[\mathrm{~K}^{+}\right]_{\mathrm{e}}$, producing RMPs of -10 to $0 \mathrm{mV}$ ) (Rosen et al., 1994; Baldassa et al., 2003). PKA was also activated by depolarization, but without any difference between Naive and SCI groups (Fig. 3C). Depolarization from RMP (usually between -65 and $-55 \mathrm{mV}$ ) to $-45 \mathrm{mV}$ is modest relative to normal AP threshold $(\sim-35 \mathrm{mV})$ in nociceptors from naive rats, and it rarely evokes APs in these neurons (Odem et al., 2018). Importantly, after SCI, $-45 \mathrm{mV}$ is a sufficiently depolarized RMP for large DSFs to reach the reduced AP threshold and generate SA (Odem et al., 2018). Using IB4 binding to distinguish between neuronal subgroups, our results show that the increased ERK 
A

B

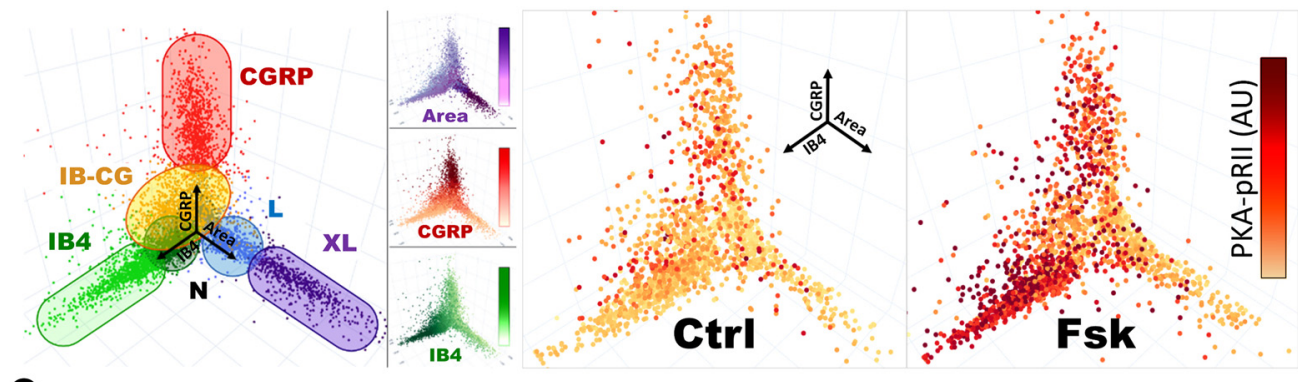

C

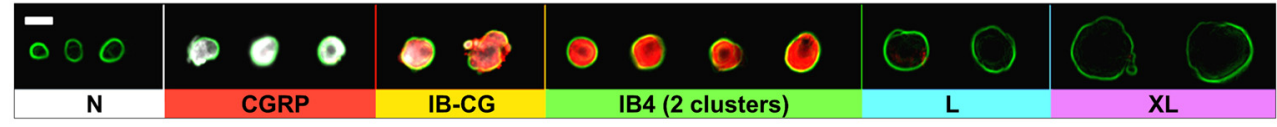

D

$\mathbf{E}$
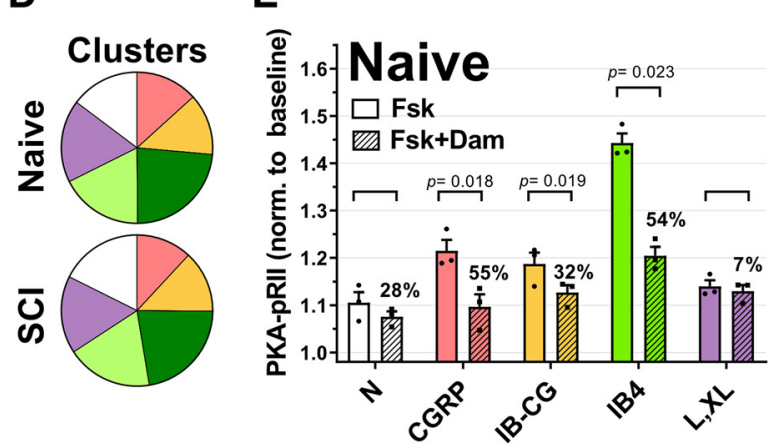

$\mathbf{F}$

Figure 2. Cluster analysis of DRG neurons. $A, 3 D$ representation of DRG neurons in culture (7638 neurons) using area ( $X)$, CGRP ( () , and IB4 (Z) values as spatial coordinates. Different colors represent the seven different clusters identified by $k$-medians cluster analysis and plotted based on $X, Y, Z$ coordinates. Neurons from two different IB4 ${ }^{+}$clusters are represented as a single group. $\boldsymbol{B}$, Representative experiment showing the neuronal subgroup specificity of PKA-pRII responses to Fsk in cultured neurons from a naive animal. Dot color saturation is proportional to the intensity of PKA-pRII fluorescent values: Darker colors represent stronger PKA-pRII signals ( $n>2000$ neurons per condition) $C$, Representative examples of DRG neurons arranged according to the identified clusters in $\boldsymbol{A}$. GGRP (white), IB4 (red), and soma size (indicated by green outline of cell membrane based on PGP9.5). Clusters include N (neurons negative for IB4 and CGRP, with small soma size), CGRP, IB-CG (weak staining for both IB4 and (GRP), IB4, and L and XL (large and extra-large soma size). Scale bar, $25 \mu \mathrm{m}$. D, Relative mean cluster size in the total neuronal population for naive and $\mathrm{SCl}$ cultures. Colors correspond to clusters in $\boldsymbol{A}$ and $\boldsymbol{C}$. Light and dark green represent weak and strong IB4 staining clusters, respectively. No significant differences in relative cluster size were found between Naive and SCI groups ( $p>0.99, n=3$ per group, two-way ANOVA, followed by Sidak's test). $E$, $F$, Fsk responses and DAMG0 effects in specific neuronal clusters for naive $(\boldsymbol{E})$ and $S C I(F)$ cultures. $n=3$ per group; DAMGO effects per cluster tested via paired $t$ test. The \% DAMGO inhibition over the control Fsk response is indicated for each group. "Red represents significance difference in DAMG0 effects comparing IB-CG and IB4 ${ }^{+}$clusters from naive $(\boldsymbol{E})$ and SCI $(\boldsymbol{F})(n=3$, unpaired $t$ test). Detailed statistical information is provided in Table 1.

activation by modest depolarization after SCI is specific to $\mathrm{IB}^{+}$neurons $(p=0.047$, two-way ANOVA), with no significant difference between Naive and SCI groups for IB4 ${ }^{-}$neurons (Fig. 3D).

\section{Depolarization-induced reduction of opioid sensitivity requires signaling by $\mathrm{C}$-Raf}

To demonstrate the predicted link between depolarized RMP in nociceptors produced by SCI and reductions in opioid sensitivity and associated ERK signaling, we tested the influence of RMP on opioid sensitivity. Neurons from the uninjured Naive group were exposed to $15 \mathrm{~mm}\left[\mathrm{~K}^{+}\right]_{\mathrm{e}}$ alone or concurrently with the 5 min Fsk or Fsk + DAMGO stimulation (Fig. 3E). RMP depolarized from $\sim-65 \mathrm{mV}$ to $\sim-45 \mathrm{mV}$ during the stimulation (Fig. $3 A$ ), reaching the optimal range of RMP for generation of large DSFs and sustained OA after SCI (Odem et al., 2018). This modest depolarization gave rise to a small, $\sim 20 \%$, increase in basal cAMP/PKA-pRII responses, consistent with the low expression of AC1/8 in DRGs (Bavencoffe et al., 2016; Dessauer et al., 2017). Overall Fsk responses were unchanged by SCI (Fig. $1 B$ ) or by 15 $\mathrm{mm}\left[\mathrm{K}^{+}\right]_{\mathrm{e}}$ in the Naive group. Under these conditions, depolarization had a striking effect on DAMGO sensitivity, dramatically decreasing DAMGO inhibition of Fsk responses compared with $5 \mathrm{~mm}\left[\mathrm{~K}^{+}\right]_{\mathrm{e}}$ control (Fig. $3 E ; p=0.0015$, two-way ANOVA), resulting in a 1.5 -fold increased $\mathrm{IC}_{50}$ and $\sim 50 \%$ decreased efficacy $\left(\mathrm{E}_{\max }\right)$ for DAMGO compared with $5 \mathrm{~mm}\left[\mathrm{~K}^{+}\right]_{\mathrm{e}}$ control (Fig. 3F; $p=4 \times 10^{-29}$, two-way ANOVA; quantitated further in Fig. $4 C, D)$. Depolarization also decreased overall inhibition of Fsk responses by morphine, a nonpeptidic MOR agonist, compared with control (Fig. $3 G ; p=0.0009$, two-way ANOVA), resulting in a sevenfold increase in the $\mathrm{IC}_{50}$ (Fig. $3 G$ ). The reduced DAMGO efficacy is likely at the level of MOR, as the reduction in $\mathrm{E}_{\max }$ is not observed with the partial agonist morphine (Fig. $3 F, G$ ), and is similar to the voltage-dependent reduction in efficacy observed with other GPCRs (Vickery et al., 2016). Moreover, regulation of MOR by voltage was recently reported (Ruland et al., 2020).

The rapid onset of $\left[\mathrm{K}^{+}\right]_{\mathrm{e}}$-induced effects (within $5 \mathrm{~min}$ ) indicates that, under these testing conditions, the observed effects are independent of transcription and/or translation and point toward direct signaling events triggered by depolarization. To determine which signaling pathways are required for the depolarization-induced shift in DAMGO responses, DRG neurons were pretreated with inhibitors of Src, PKC, PKA, Raf, and MEK 
A

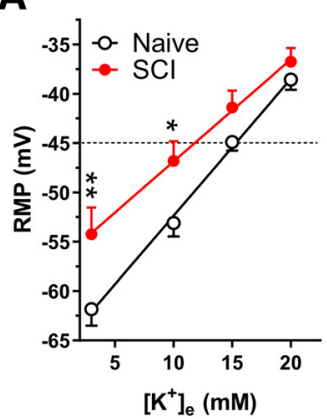

E

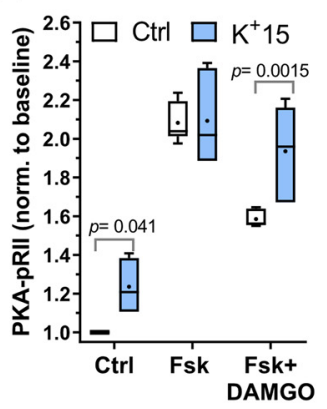

B

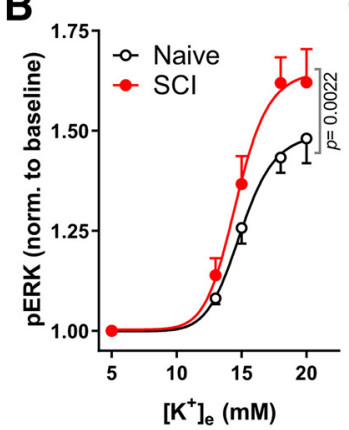

F

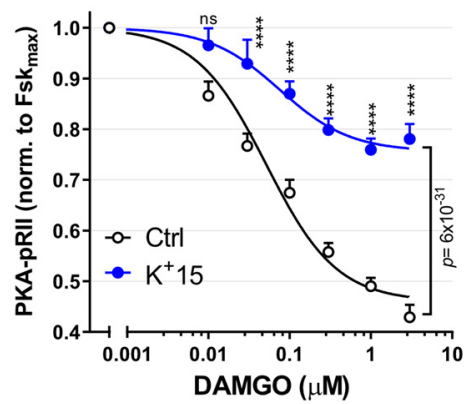

C

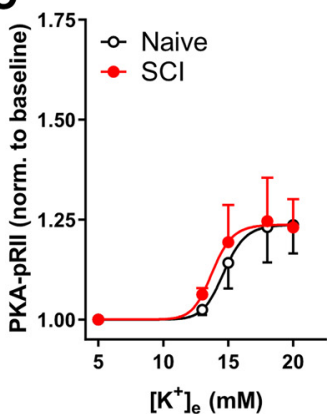

G
D

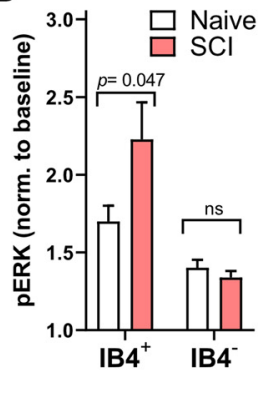

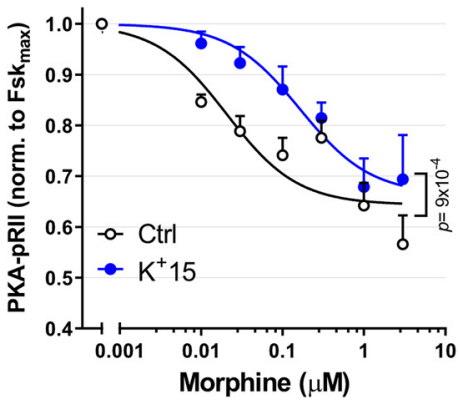

Figure 3. Acute depolarization activates ERK and reduces DAMGO effects on cAMP. A, RMP for Naive (black) and SCI (red) small- to medium-sized DRG neurons were measured on successive perfusions (30 s) of increasing extracellular $\mathrm{K}^{+}$concentration, $\left[\mathrm{K}^{+}\right]_{\mathrm{e}}$. RMP at each $\left[\mathrm{K}^{+}\right]_{\mathrm{e}}$ was measured when steady state was reached $(n=11)$. ${ }^{*} p=0.039 ;{ }^{* *} p=0.0081$; two-way ANOVA with Sidak's test. $\boldsymbol{B}, \boldsymbol{C}$, Concentration-response curves for pERK $(\boldsymbol{B})$ and PKA-pRII $(\boldsymbol{C})$ to increased $\left[\mathrm{K}^{+}\right]_{\mathrm{e}}$. Neurons were exposed to media with indicated $\left[\mathrm{K}^{+}\right]_{\mathrm{e}}$ for 5 min. $\boldsymbol{B}$, Naive, $n=5-7$; $\boldsymbol{S C l}$,

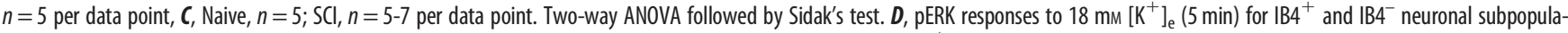
tions in Naive and SCI DRG neurons: Naive, $\mathrm{SCl}, n=3, p=0.047$, two-way ANOVA followed by Sidak's test. $\boldsymbol{E}, \mathrm{K}^{+}$-induced depolarization decreases DAMGO responses in Naive DRG neurons. Neurons were exposed to either control media $\left(5 \mathrm{~mm}\left[\mathrm{~K}^{+}\right]_{\mathrm{e}}\right)$ or $15 \mathrm{~mm}\left[\mathrm{~K}^{+}\right]_{\mathrm{e}}$ media during Fsk \pm DAMGO (Dm) stimulation (Fsk $\left.=3 \mu \mathrm{m}, \mathrm{DAMGO}=1 \mu \mathrm{M}, 5 \mathrm{~min}\right) ; n=6$. Control, $p=0.041$; Fsk +DAMG0, $p=0.0015$, two-way ANOVA, followed by Sidak's test. Box-and-whisker plot represents the median, mean $(+)$, quartiles, and range of the data. $\boldsymbol{F}$, Dose-response curves of DAMG0 inhibition of Fsk responses in Naive DRG neurons under control conditions (black) or $15 \mathrm{~mm}\left[\mathrm{~K}^{+}\right]_{\mathrm{e}}$ (blue). I I ${ }_{50}$ Control, $0.051 \mu \mathrm{m} ; \mathrm{IC}_{50} \mathrm{~K}^{+} 15,0.075 \mu \mathrm{m}$; Control, $n=9-19 ; \mathrm{K}^{+} 15, n=4-$ 12 per data point. Data compared by two-way ANOVA, followed by Sidak's test: ${ }^{* * *} p<0.0001$. G, Dose-response curves of morphine inhibition of Fsk responses in naive DRG neurons with control or $15 \mathrm{~mm}\left[\mathrm{~K}^{+}\right]_{\mathrm{e}}$. Control IC $\mathrm{I}_{50}=0.021 \mu \mathrm{m} ; \mathrm{K}^{+} 15 \mathrm{IC}_{50}=0.16 \mu \mathrm{m}$; Control, $n=3-5 ; \mathrm{K}^{+} 15, n=4$ or 5 per data point (two-way ANOVA). Detailed statistical information is provided in Table 1.

for $30 \mathrm{~min}$ to test for their ability to block reductions in DAMGO responses by $15 \mathrm{~mm}\left[\mathrm{~K}^{+}\right]_{\mathrm{e}}$. Selective C-Raf (GW5074) and pan-Raf (RAF709) inhibitors blocked the effects of $5 \mathrm{~min}$ depolarization when tested with $0.1 \mu \mathrm{M}$ DAMGO (Fig. 4A; $p=0.0082$, one-way ANOVA). Interestingly, the C-Raf inhibition ( $3 \mu \mathrm{m}, 30 \mathrm{~min}$ ) appeared to reverse the increase in DAMGO $\mathrm{IC}_{50}$ induced by $15 \mathrm{~mm}\left[\mathrm{~K}^{+}\right]_{\mathrm{e}}$ (Fig. $4 B, C$; Control vs $\mathrm{K}^{+} 15(-)$ : $p=0.013 ; \mathrm{K}^{+} 15(-)$ vs $\mathrm{K}^{+} 15(\mathrm{GW}): p=0.034$, one-way ANOVA), but not the alteration in DAMGO $\mathrm{E}_{\max }$ (Fig. $4 B, D$; Control vs $\mathrm{K}^{+} 15(-): p=3 \times 10^{-8}$, one-way ANOVA). In contrast, under control conditions $\left(5 \mathrm{~mm}\left[\mathrm{~K}^{+}\right] \mathrm{e}\right)$, GW5074 pretreatment had no effects on DAMGO sensitivity (Fig. $4 B$, dotted line). The lack of effects by inhibitors of MEK or PKC (UO126 and sotrastaurin, respectively), suggests that signaling previously reported to modulate MOR responses via receptor desensitization (ERK) (Polakiewicz et al., 1998) or phosphorylation of G $\alpha$ i2 (PKC) (Strassheim and Malbon, 1994) are not required for depolarization-induced reductions in DAMGO sensitivity.

Raf activation is complex and involves several mechanisms, including but not limited to Ras-mediated recruitment of Raf to the cell membrane followed by multiple phosphorylations via different protein kinases that enhance C-Raf catalytic activity (S338 among the best studied) (for review, see Leicht et al., 2007). In addition, C-Raf signaling requires relief of inhibition by RKIP via the phosphorylation of RKIP-S153 (Fig. 5A) (Corbit et al., 2003). To examine which of these mechanisms are triggered by depolarization of nociceptors, we examined the effect of
$\left[\mathrm{K}^{+}\right]_{\mathrm{e}}$-induced depolarization (5 min) on RKIP-S153 and C-RafS338 phosphorylation in the total DRG neuronal population (Fig. 5B). Depolarization triggered a rapid, dose-dependent increase in C-Raf and RKIP phosphorylation, rising steeply as a function of $\left[\mathrm{K}^{+}\right]_{\mathrm{e}}$ with an $\mathrm{EC}_{50}$ of $14.72 \pm 0.40 \mathrm{mM}$ for $\mathrm{pC}$-Raf and $13.11 \pm 0.41 \mathrm{mM}$ for pRKIP (corresponding to a RMP of $\sim-46 \mathrm{mV}$ and $-48 \mathrm{mV}$ for $\mathrm{pC}$-Raf and pRKIP, respectively). As observed for pERK, $\left[\mathrm{K}^{+}\right]_{\mathrm{e}} 15 \mathrm{~mm}$ induced significantly higher phosphorylation of C-Raf in the SCI group (Fig. $5 C ; p=0.038$, unpaired $t$ test). Depolarization-induced phosphorylation of CRaf and RKIP occurred in several types of DRG neurons, with the highest increases detected in the small soma size, $\mathrm{IB} 4^{+}$group (Fig. 5D,E; Fig. 5F, bottom left quadrant of the 3D cluster maps; Fig. 5G). C-Raf directly phosphorylates and regulates $\mathrm{G} \alpha \mathrm{i}$-sensitive AC isoforms (Tan et al., 2001; Ding et al., 2004). Therefore, to demonstrate that C-Raf activation is sufficient to decrease DAMGO sensitivity, we expressed a membrane-targeted active C-Raf construct in HEK293 cells stably expressing the MOR (Fig. 6). Transient expression of plasma membrane-targeted CRaf gave rise to a 2.2-fold shift in the $\mathrm{IC}_{50}$ for DAMGO (Fig. $6 \mathrm{~B}$; $p=0.0028$, one-way ANOVA) with no alterations in the $\mathrm{E}_{\max }$ (Fig. 6A,C). Serum treatment had similar effects, producing a 2.8 -fold shift in the DAMGO IC I0 $_{5}(p=0.016$, one-way ANOVA) that was reversed by the C-Raf inhibitor GW5074 ( $p=0.026$, one-way ANOVA). Interestingly, while treatment of nonexcitable HEK293 cells with 15 and $50 \mathrm{~mm}\left[\mathrm{~K}^{+}\right]_{\mathrm{e}}$ did not alter the $\mathrm{IC}_{50}$ of DAMGO or induce activation of C-Raf (Fig. $6 \mathrm{~B}$; and data 
A
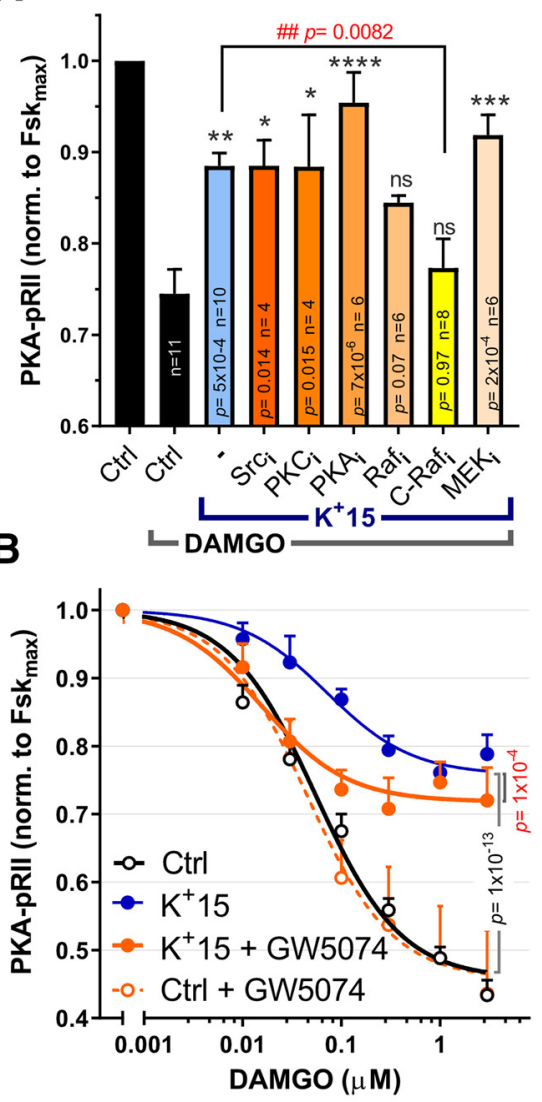

C

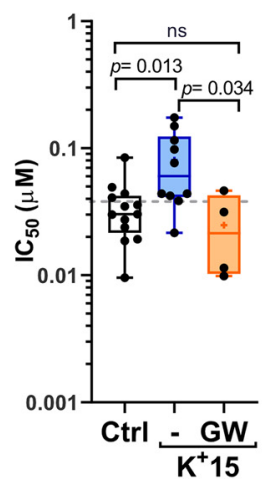

D

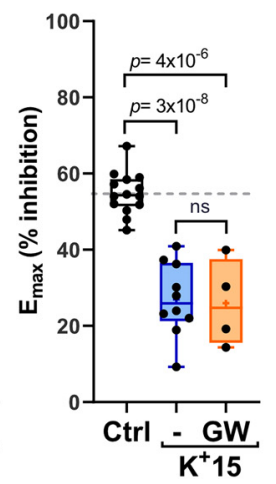

Figure 4. Inhibition of C-Raf partially restores opioid sensitivity after depolarization. $\boldsymbol{A}$, Inhibition of $15 \mathrm{~mm}\left[\mathrm{~K}^{+}\right]_{\mathrm{e}}$ effects on DAMGO responses. DRG neurons were preincubated with inhibitors of Src (saracatinib, $10 \mu \mathrm{m})$, PKC (sotrastaurin, $1 \mu \mathrm{m})$, PKA (H89, $10 \mu \mathrm{m}), \mathrm{C} / \mathrm{B}$ Raf (RAF709, $10 \mu \mathrm{m})$, C-Raf (GW5074, $3 \mu \mathrm{m})$, or MEK1/2 (U0126, $10 \mu \mathrm{m})$ for $30 \mathrm{~min}$ and then stimulated with Fsk $(3 \mu \mathrm{m}) \pm \operatorname{DAMGO}(0.1 \mu \mathrm{m})$ in control media or $15 \mathrm{~mm}\left[\mathrm{~K}^{+}\right]_{\mathrm{e}}$ $\left(\mathrm{K}^{+} 15 ; 5 \mathrm{~min}\right)$. Effects are reported as inhibition of the control Fsk response (1.0). Drug effects were compared against the Control-DAMGO inhibition $\left(^{*}\right)$ or $\mathrm{K}^{+}$-DAMGO inhibition (\#) via one-way ANOVA, followed by Dunnett's test. Significance and $n$ values are reported on each bar. ${ }^{*} p<0.05,{ }^{* *} p<0.01,{ }^{* *} p<0.001,{ }^{* * *} p<0.0001,{ }^{* \#} p<0.01$. B, C-Raf inhibition restores DAMGO IC 50 in $15 \mathrm{~mm}\left[\mathrm{~K}^{+}\right]_{\mathrm{e}}$, but has no effect on DAMGO efficacy ( $\left.E_{\max }\right)$. GW5074 $(10 \mu \mathrm{m})$ was applied 30 min before simultaneous addition of $\mathrm{K}^{+}(15 \mathrm{mM})$, Fsk (3 mM), and DAMG0 for $5 \mathrm{~min}$. Per data point, Control, $n=10-21 ; \mathrm{K}^{+} 15, n=6-22$; $\mathrm{K}^{+}$15+GW5074, $n=3-10$; Control+GW5074, $n=3-11$. Treatment effects were compared using two-way ANOVA followed by Tukey's multiple comparisons test. Individual data point comparisons using Sidak's test can be found in Table 1. C, D, Quantification of individual I $\boldsymbol{C}_{50}$ $(\boldsymbol{C})$ and $E_{\max }(\boldsymbol{D})$ values of DAMGO dose-response curves as described in $\boldsymbol{B}$. C, Control, $n=13 ; \mathrm{K}^{+} 15(-), n=10 ; \mathrm{K}^{+} 15(\mathrm{GW}), n=4 . \boldsymbol{D}$, Control, $n=14 ; \mathrm{K}^{+} 15(-), n=10 ; \mathrm{K}^{+} 15$ $(\mathrm{GW}), n=4$. Data were compared using one-way ANOVA, followed by Tukey's test. Box-andwhisker plot represents the median, mean $(+)$, quartiles, and range of the data. Detailed statistical information is provided in Table 1. not shown), $\left[\mathrm{K}^{+}\right]_{\mathrm{e}}$ dose-dependently reduced the $\mathrm{E}_{\max }$ of DAMGO ( $10 \%$ and $47 \%$ reduction in $\mathrm{E}_{\max }$, respectively; $p=4 \times$ $10^{-4}$ and $p<0.0001$, one-way ANOVA) (Fig. 6C). Thus, treatment with $\left[\mathrm{K}^{+}\right]_{e}$ requires C-Raf activation to alter DAMGO potency, while the effects of depolarization on DAMGO $\mathrm{E}_{\max }$ may indeed indicate a voltage-sensitive mechanism acting directly on the MOR (Vickery et al., 2016; Ruland et al., 2020).

\section{Reduction of opioid sensitivity and production of hyperexcitability by SCI are reversed by treatments that inhibit C-Raf signaling or hyperpolarize RMP}

We next tested whether inhibition of C-Raf or reversing the SCImediated depolarization of nociceptors is sufficient to restore DAMGO responses after SCI. Inhibition of C-Raf with the selective C-Raf inhibitor GW5074 restored the $\mathrm{IC}_{50}$ of DAMGO in nociceptors from SCI rats to that found in the Naive group (Fig. $7 A$ ). A clinically relevant approach to reversing depolarization is to open $\mathrm{K}^{+}$channels pharmacologically. KCNQ (M-type) channels contribute $\sim 9 \mathrm{mV}$ to the RMP of capsaicin-sensitive nociceptors, and application of the KCNQ channel opener, retigabine $(10 \mu \mathrm{M})$ is reported to produce an additional $10 \mathrm{mV}$ hyperpolarization of RMP (Du et al., 2014). Moreover, $10 \mu \mathrm{M}$ retigabine was shown to hyperpolarize small DRG neurons isolated from SCI rats (Wu et al., 2017). We found that this dose of retigabine restored the $\mathrm{IC}_{50}$ of DAMGO for the SCI group to a value close to that of the Naive group (Figs. $1 D, 7 A, B ; \mathrm{IC}_{50}$, Naive $=0.053 \pm 0.006$ and retigabine $=0.023 \pm 0.009$ ). Hyperexcitability of nociceptors induced by SCI is manifested not only as depolarized RMP, but also as SA at RMP in vivo and in vitro (Bedi et al., 2010) and enhanced OA revealed during prolonged experimental depolarization to $-45 \mathrm{mV}$ (Odem et al., 2018). Pretreatment of neurons from SCI rats with inhibitors of C-Raf (GW5074) or MEK (UO126) blocked SA and OA and led to a hyperpolarization of RMP (Fig. 7C-E; Control $=-53 \pm 2 \mathrm{mV}$, GW5074 $=-62 \pm 2 \mathrm{mV}$, UO126 $=-62 \pm 3 \mathrm{mV}$ ), whereas pretreatment of DRG neurons from naive rats with UO126 had no effect on SA, OA, or RMP (data not shown; RMP Control = $-66 \pm 2 \mathrm{mV}, n=14 ; \mathrm{UO} 126=-65 \pm 2 \mathrm{mV}, n=9$; GW5074 = $-60 \pm 3 \mathrm{mV}, n=8$; Kruskal-Wallis with Dunn's multiple comparison, $p=0.14$ ). Inhibition of $\mathrm{C}$-Raf and MEK also reduced another functional contribution to SCI-induced hyperactivity in nociceptors: the enhancement of DSFs of membrane potential that bridge the gap between RMP and AP voltage threshold to trigger APs (Odem et al., 2018). The mean amplitude of DSFs (subthreshold and suprathreshold) was significantly reduced when treated with GW5074 or UO126 and assayed at a holding potential of $-45 \mathrm{mV}$ (Fig. $7 F$ ), as was the amplitude of the subthreshold DSFs alone $(p=0.015$ for GW5074, $p=0.013$ for UO126, Kruskal-Wallis with Dunn's multiple comparison; data not shown). DSF amplitudes were not significantly reduced when measured at RMP, at least in part because DSFs are voltage-dependent (Odem et al., 2018) and RMPs are quite variable (Fig. 7E). When tested on nociceptors from SCI, only UO126 increased the rheobase (data not shown in figure: vehicle = $35 \pm 7 \mathrm{pA} ; \mathrm{GW} 5074=57 \pm 16 \mathrm{pA}$, not significant; UO126 = $83 \pm 14 \mathrm{pA}, p=0.006$, Kruskal-Wallis with Dunn's multiple comparison), while neither drug had a significant effect on AP threshold (data not shown; Brown-Forsythe and Welsh Tests). C-Raf/ERK and CAMP pathways are thus both required to maintain the increased excitability after SCI (Fig. 7D-F) (Bavencoffe et al., 2016; Berkey et al., 2020). C-Raf activation of its canonical downstream target, MEK, produces an ERK-dependent positive feedback loop that helps to maintain increased SA and 
A

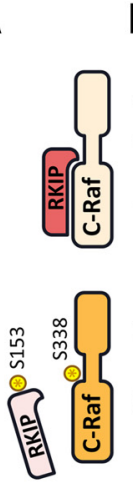

B

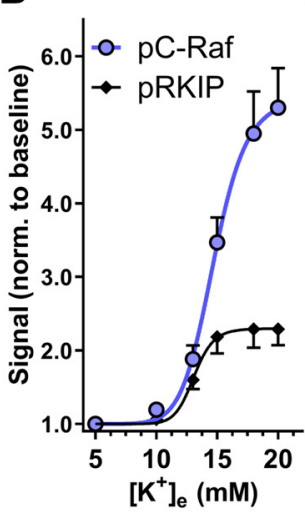

$\mathbf{F}$

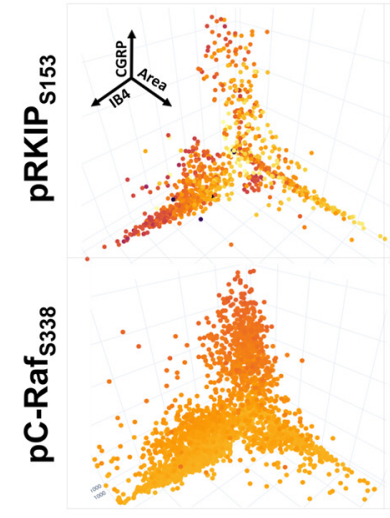

Control
C
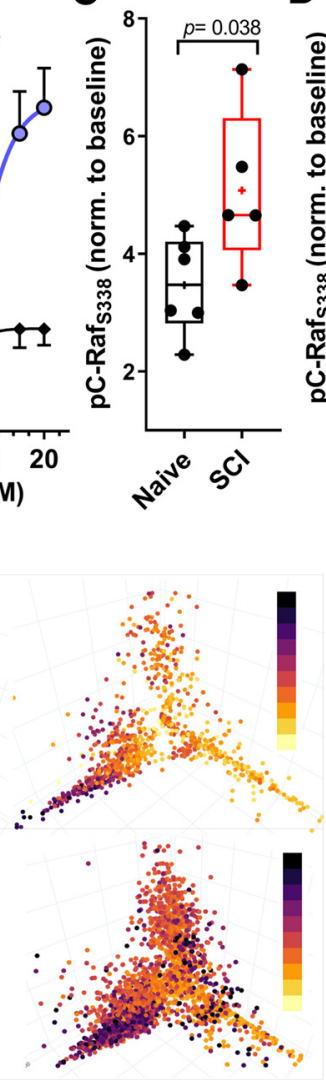

$\left[\mathrm{K}^{+}\right]_{\mathrm{e}} 15 \mathrm{mM}$
D

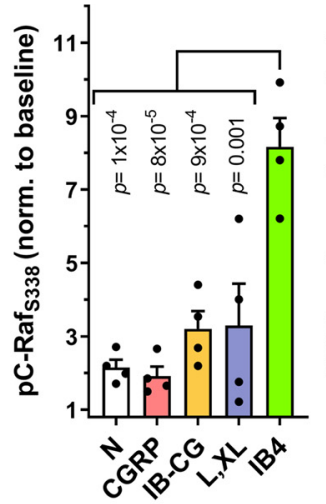

E

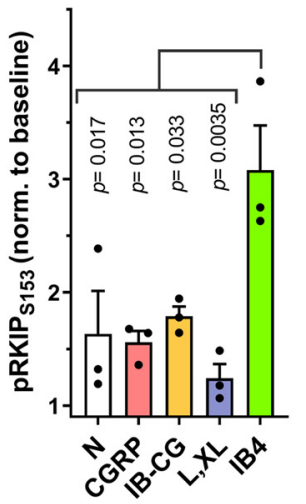

G

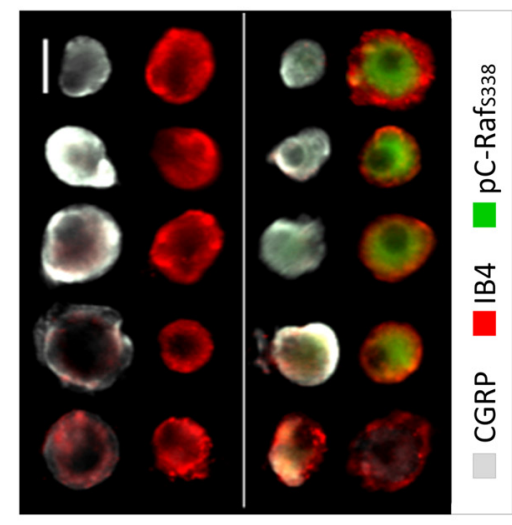

Control $\left[\mathrm{K}^{+}\right]_{\mathrm{e}} 15 \mathrm{mM}$

Figure 5. Depolarization induces C-Raf activation and relief of RKIP inhibition in IB4 ${ }^{+}$neurons. $A, C$-Raf activity is promoted by phosphorylation (S338). C-Raf inhibition is relieved on phosphorylation of RKIP (S153). B, Phosphorylation of C-Raf (S338) and RKIP (S153) in response to increasing $\left[K^{+}\right]_{e}$ (5 min stimulation). pC-Raf $5_{5338}, n=3-7 ;$ pRKIP S153, $_{n} n=3$. C, Phosphorylation of C-Raf (S338) with $15 \mathrm{~mm}\left[\mathrm{~K}^{+}\right]_{\mathrm{e}}$ in naive and SCI DRG neurons; Naive, $n=6 ; S C \mathrm{I}, n=5$. Data compared via unpaired $t$ test. Box-and-whisker plot represents the median, mean $(+)$, quartiles, and range of the data. $\boldsymbol{D}, \boldsymbol{E}$, Quantification of pC-Raf $\mathrm{S}_{338}(\boldsymbol{D}, n=4)$ and pRKIP ${ }_{5153}(\boldsymbol{E}, n=3)$ phosphorylation in naive neurons in response to $5 \mathrm{~min}, 15 \mathrm{~mm}\left[\mathrm{~K}^{+}\right]_{\mathrm{e}}$ between different neuronal subpopulations (clus-

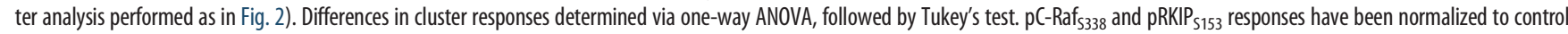
baselines for each cluster. $p$ values in the figure correspond to the comparison against IB4. Detailed statistical information is provided in Table 1. F, C-Raf and RKIP phosphorylation in control and 15 $\mathrm{mm}\left[\mathrm{K}^{+}\right]_{\mathrm{e}}$, shown as coordinates of nociceptor Area (X), CGRP ( () , and IB4 (Z) intensity. Dot color saturation is proportional to the intensity of fluorescent signal. Darker colors represent stronger signals. pRKIP: $n>1000$ neurons; pC-Raf: $n>3200$ neurons. G, Examples of pC-Raf 5338 responses to depolarization $\left.\left(5 \mathrm{~min}, 15 \mathrm{~mm}^{+} \mathrm{K}^{+}\right]_{\mathrm{e}}\right) .10 \times$ magnification. Scale bar, $25 \mu \mathrm{m}$.

A

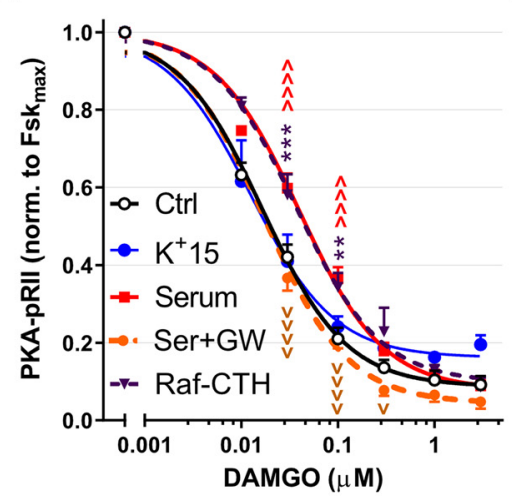

B

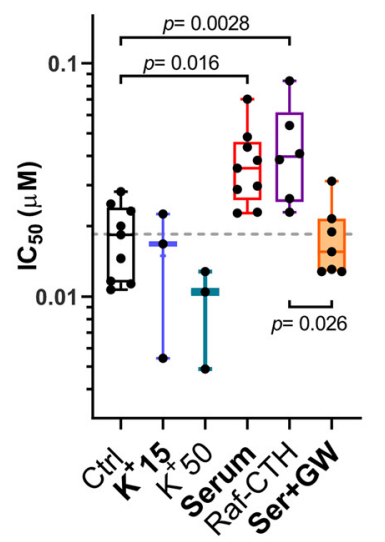

C

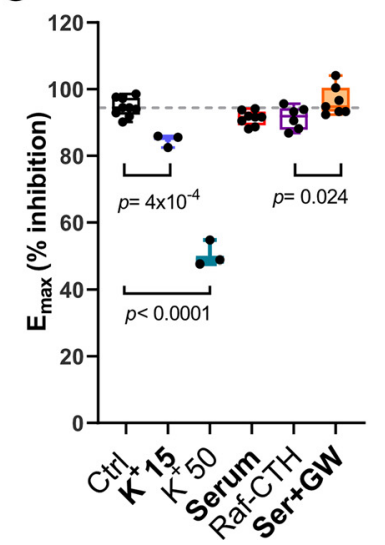

Figure 6. Effects of depolarization and C-Raf activation on HEK-293MOR cells. A, DAMGO dose-response curves in control conditions ([K $\left.{ }^{+}\right]_{\mathrm{e}} 5 \mathrm{~mm}$, serum-starved; black empty circles), $\left[\mathrm{K}^{+}\right]_{\mathrm{e}}$ $15 \mathrm{~mm}$ (blue circles), (-Raf-CTH overexpression (Raf-CTH; purple triangles, dotted line), serum treatment (not starved, red squares), or serum in presence of $3 \mu \mathrm{m}$ the C-Raf inhibitor GW5074 (Ser+GW; orange circles, dotted line). Control, $n=7-9 ; \mathrm{K}^{+} 15, n=3$; Serum, $n=3-11$; Raf-CTH, $n=6$; Ser $+\mathrm{GW}, n=7$ or 8 per data point. Treatment effects were compared via two-way ANOVA, followed by Sidak's test. ${ }^{\vee} p<0.05 ;{ }^{* *} p<0.01 ;{ }^{* * *} p<0.001 ;{ }^{\wedge \wedge \wedge \wedge, \text { vwv }} p<0.0001 .{ }^{*}$ Control versus Raf-CTH. ${ }^{\wedge}$ Control versus Serum. " Serum versus Ser $+G W$. B, C, Effects of the different treatments used in $A$, on DAMGO IC 50 (B) and $\mathrm{E}_{\max }(\boldsymbol{C}) ; 50 \mathrm{~mm} \mathrm{~K} \mathrm{~K}^{+}$not shown in $\boldsymbol{A}$. $\boldsymbol{B}$, Control, $n=9 ; \mathrm{K}^{+} 15, n=3 ; \mathrm{K}^{+} 50, n=3 ;$ Ser, $n=9 ;$; Raf-CTH, $n=6$; Ser + GW, $n=7$. C, Control, $n=9 ; \mathrm{K}^{+} 15, n=3 ; \mathrm{K}^{+} 50, n=3$; Ser, $n=8$; Raf-CTH, $n=6$; Ser $+\mathrm{GW}, n=7$. Treatment effects compared via one-way ANOVA followed by Dunnett's test. Box-and-whisker plot represents the median, mean $(+)$, quartiles, and range of the data. Detailed statistical information is provided in Table 1. 
A

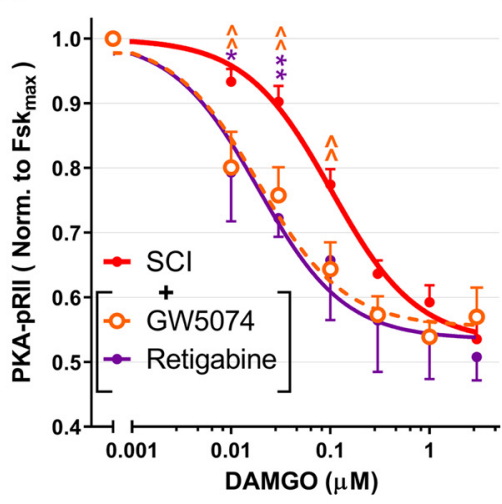

D

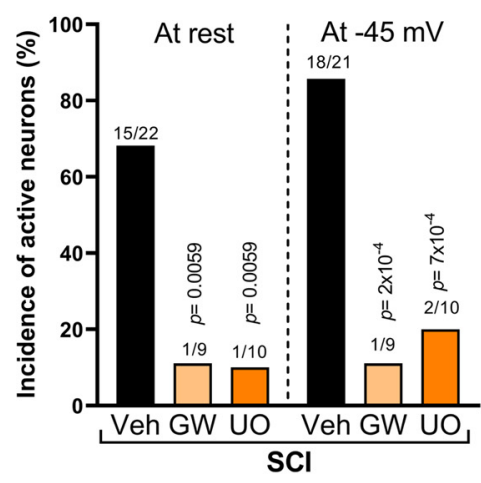

B

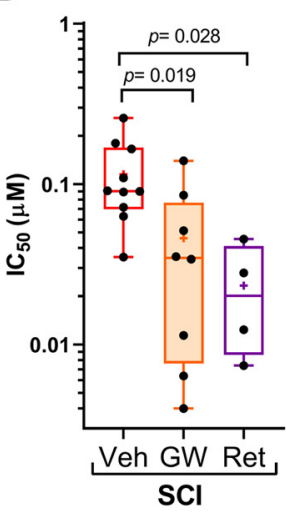

E

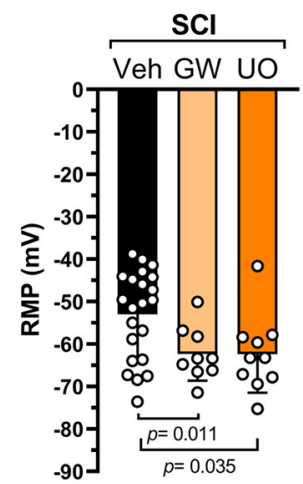

C

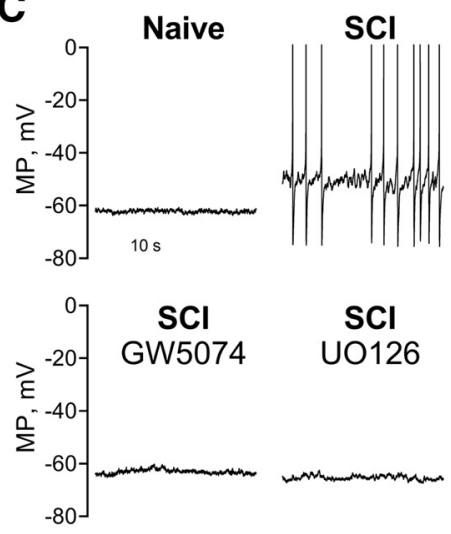

$\mathbf{F}$

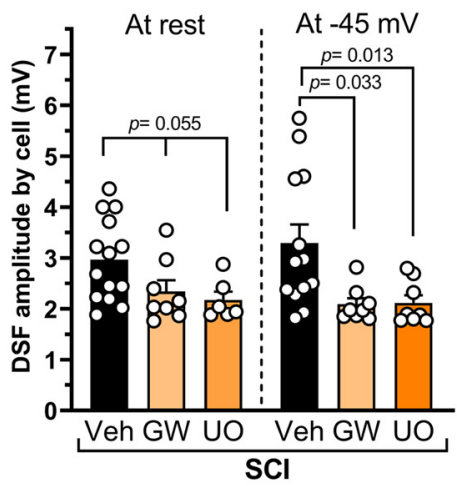

Figure 7. Inhibition of C-Raf fully restores opioid sensitivity and reverses hyperexcitability after depolarization. A, DAMGO inhibition of Fsk responses in $\mathrm{SCl}$ cultured neurons pretreated for 30 min with vehicle (DMSO $\leq 0.1 \%)$, GW5074 $(3 \mu \mathrm{m})$, or retigabine $(10 \mu \mathrm{m})$, followed by 5 min stimulation with Fsk $(3 \mu \mathrm{m})+/-$ indicated concentrations of DAMG0. Vehicle, $n=11$; GW5074, $n=7$ or 8 ; retigabine, $n=4$. Treatment effects compared via two-way ANOVA, followed by Sidak's test. ${ }^{*} p<0.05$. ${ }^{* *}, \wedge p<0.01$. Significance symbols: *Vehicle versus retigabine. ${ }^{\wedge}$ Vehicle versus GW5074. B, IC $C_{50}$ values from individual DAMGO dose-response curves for SCI groups treated as in $\boldsymbol{A}$. Veh, $n=10 ; \mathrm{GW}, n=8$; Ret, $n=4$. Treatment effects compared using one-way ANOVA, followed by Dunnett's test. Box-and-whisker plot represents the median, mean (+), quartiles, and range of the data. C, Representative traces of current-clamp recordings $(I=0)$ of sensory neurons isolated from Naive and SCI groups pretreated (30 min) with vehicle (DMSO 0.03\%), C-Raf inhibitor (GW5074, $3 \mu \mathrm{m})$ or MEK inhibitor (U0126, $3 \mu \mathrm{M})$. APs are clipped at $0 \mathrm{mV}$ so that subthreshold DSFs are more visible. D, A selective C-Raf inhibitor (GW5074) and MEK inhibitor (U0126) decrease the incidence (\%) of neurons exhibiting ongoing firing at RMP $(\mathrm{SA})$ or when held at $-45 \mathrm{mV}(\mathrm{OA})$. Comparisons of active neuron incidence by Fisher's exact test. $n$ values and $p$ values are indicated over each bar. For multiple comparisons, Bonferroni correction was applied and significance levels are provided on figure. $E$, Inhibition of C-Raf or MEK by GW5074 or U0126, respectively, hyperpolarizes RMP. The effects of C-Raf and MEK inhibitors on RMP were compared with Brown-Forsythe and Welch ANOVA, followed by Dunnett's test ( $p=0.011$ for GW5074 and $p=0.035$ for U0126). $\boldsymbol{F}$, Reduction of DSF amplitudes by GW5074 and U0126. Mean DSF amplitudes trended lower when recorded at RMP ( $p=0.055$ for each inhibitor) and were significantly lower at $-45 \mathrm{mV}(p=0.033$ for GW5074 and $p=0.013$ for U0126). One-way ANOVA followed by Holm-Sidak's test at RMP and Kruskal-Wallis with Dunn's multiple comparison test at $-45 \mathrm{mV}$. MP, Membrane potential. Detailed statistical information is provided in Table 1.

depolarized RMP chronically after SCI. In addition, C-Raf effects on AC (independent of MEK-ERK activation) promote cAMP signaling by decreasing its inhibition from opioid/G $\alpha$ i signaling (Fig. 8). When depolarization is produced by elevating extracellular $\mathrm{K}^{+}$(i.e., without the need for any cell signaling input), inhibition of ERK has no direct effect on opioid responsiveness. This finding suggests that the activation of C-Raf by SCI-induced depolarization of RMP (and perhaps other manifestations of hyperexcitability) triggers two independent signaling pathways acting in parallel to maintain the nociceptor hyperexcitable state (Fig. 8).

\section{Discussion}

Previously, we showed that SCI causes a profound reduction of G $\alpha$ i-mediated inhibition of AC activity independent of endogenous receptors or $\mathrm{G}$ proteins, as revealed by comparing purified G protein-stimulated DRG membranes from naive and SCI rats (Bavencoffe et al., 2016). The present study shows that in vitro SCI-induced reduction of G $\alpha$ i inhibition (1) also occurs in intact cell bodies of DRG neurons in dissociated cell culture, (2) is

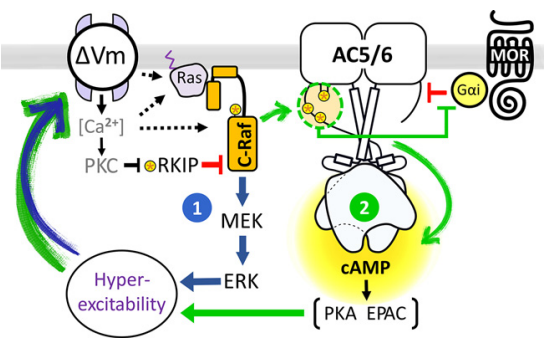

Figure 8. Model of depolarization-dependent, C-Raf-mediated, self-reinforcing mechanisms driving nociceptor hyperexcitability and reduced opioid responses after $\mathrm{SCl}$. Depolarization of nociceptors $(\Delta \mathrm{Vm})$ induced by $\mathrm{SCl}$ enhances $\mathrm{C}$-Raf activity in $\mathrm{IB}^{+}$neurons via direct phosphorylation of C-Raf and relief from RKIP inhibition. Active C-Raf promotes hyperexcitability via two different mechanisms acting in parallel. (1) Activation of the MEKERK cascade by (-Raf has direct effects on RMP and neuronal excitability. (2) Phosphorylation of AC5/6 by C-Raf reduces the inhibitory effects of G $\alpha$ i on CAMP generation by $A C$ and downstream PKA/EPAC signaling, which also regulate nociceptor hyperexcitability and RMP. The combined effects of MAPK and CAMP signaling on RMP and hyperexcitability set up positive feedback that maintains $0 A / S A$ in nociceptors while also limiting the effectiveness of opioids. 
most prominent in $\mathrm{IB}^{+}{ }^{+}$nociceptors, (3) is produced unexpectedly by modest depolarization of RMP, and (4) requires signaling through C-Raf. Moreover, while ERK activity is not required for effects on opioid insensitivity, ERK is necessary for SCI-induced nociceptor hyperexcitability. These findings provide new insight into the signaling within primary nociceptors that reduces sensitivity to endogenous and therapeutic opioids, and that may promote ongoing pain through multiple mechanisms, including a previously unappreciated signaling role for RMP.

\section{Depolarization-induced effects on MAPK and cAMP signaling}

Depolarization of RMP (typically produced experimentally by elevating $\left[\mathrm{K}^{+}\right]_{\mathrm{e}}$ ) has long been known to stimulate cellular signaling, including signaling by mitogen-activated protein kinase (MAPK) cascades (Rosen et al., 1994). Depolarization-induced signaling is often assumed to be mediated by increases in cytoplasmic $\mathrm{Ca}^{2+}$ because of activation of voltage-gated $\mathrm{Ca}^{2+}$ channels, although other voltage-sensitive, $\mathrm{Ca}^{2+}$-independent mechanisms can contribute to ERK activity (for review, see McLaughlin and Levin, 2018). In rat DRG neurons, depolarization by $15 \mathrm{~mm}\left[\mathrm{~K}^{+}\right]_{\mathrm{e}}$ induced a rapid and robust activation of ERK and phosphorylation of its upstream regulators, RKIP and C-Raf. Observed predominantly in $\mathrm{IB}^{+}$neurons, these responses occurred at physiological RMPs that normally are subthreshold for evoking APs $(-50$ to $-40 \mathrm{mV})$. The ERK responses to depolarization were further enhanced by SCI. Importantly, $-45 \mathrm{mV}$ is within the RMP range where, after SCI, large DSFs can reach AP threshold to generate sustained spontaneous and OA (Odem et al., 2018; Berkey et al., 2020). The actual MP should be $\sim 4 \mathrm{mV}$ more negative than the values presented because our measurements were not corrected for the liquid junction potential (see Materials and Methods).

Depolarization of RMP also induced a $\sim 20 \%$ increase in basal cAMP/PKA signaling, similar to increases in cortical neurons that generate substantial cross-talk with the MAPK pathway (Baldassa et al., 2003). However, neither depolarization nor SCI altered Fsk-stimulated AC activity in DRG neurons. Forksolin induced a robust cAMP response that was inhibited by the MOR agonist DAMGO in $\mathrm{CGRP}^{+}$and $\mathrm{IB}^{+}{ }^{+} \mathrm{DRG}$ neurons from naive rats. Modest depolarization of naive DRG neurons significantly blunted DAMGO inhibition of cAMP responses in terms of both the $\mathrm{IC}_{50}$ and efficacy $\left(\mathrm{E}_{\max }\right)$ of DAMGO, by independent mechanisms. Reduction of DAMGO IC $_{50}$ by depolarization was similar to that by SCI, both requiring C-Raf activity. In contrast, depolarization effects on opioid efficacy were C-Raf independent, agonist-specific (morphine vs DAMGO), and were observed in neurons and HEK cells, suggesting a receptor-mediated mechanism. Voltage can modulate the efficacy of MOR coupling to G proteins and $\beta$-arrestin in an agonist-specific manner (Ruland et al., 2020). Altered balance of voltage-regulated G-protein/ $\beta$-arrestin activation may explain our $\mathrm{E}_{\max }$ effects at high DAMGO concentrations.

\section{Molecular model for depolarization-dependent regulation of AC activity and ERK activity that drives nociceptor hyperexcitability and opioid insensitivity after SCI}

The findings discussed above suggest a model (Fig. 8) in which persistent depolarization of RMP after SCI continuously activates the Ras/C-Raf/MEK/ERK cascade, at least in part via enhanced C-Raf phosphorylation and relief of C-Raf inhibition by RKIP. While maintenance of hyperexcitability requires concurrent ERK activation and CAMP signaling in nociceptors after SCI, activation of C-Raf is sufficient to reduce AC inhibition by opioids independent of either ERK or PKA activity. Based on our biochemical evidence from DRG membranes (Bavencoffe et al., 2016), this model assumes that chronic reduction in Goimediated inhibition of AC induced by SCI is because of a modification of $\mathrm{AC}$ or a closely associated protein that regulates $\mathrm{AC}$. $\mathrm{C}$-Raf directly phosphorylates AC5/6, but not AC1, on multiple serine residues (Ding et al., 2004) and coimmunoprecipitates with AC6 (Ding et al., 2004; Beazely et al., 2005). Moreover, CRaf phosphorylation of AC6 in HEK293 cells leads to a 50\% and twofold increase in basal and Fsk-stimulated AC6 activity, respectively. However, regulation by $\mathrm{G} \alpha \mathrm{i}$ was never tested. We show that overexpression of activated C-Raf in HEK293 cells resulted in reduced DAMGO sensitivity, but the effect was not as strong as that observed in DRG neurons. The MOR recruits CRaf via the scaffold Hint1 and facilitates phosphorylation of AC5/6 (Rodríguez-Muñoz et al., 2011; Zhang et al., 2013). Therefore, scaffolding and/or accessory proteins in DRG neurons may facilitate C-Raf regulation of AC. Alternatively, AC isoforms expressed in DRGs versus HEK293 cells may be more sensitive to C-Raf regulation. Importantly, none of our inhibitor or overexpression studies supports a role for increased MOR desensitization or internalization after SCI or depolarization in C-Raf-mediated effects. Depolarization increases $\delta$ OR insertion into the membrane but has no reported effect on MOR internalization (for review, see Zhang et al., 2015). Furthermore, peripheral MOR are upregulated, not downregulated, after contusive thoracic SCI in mice (Liu et al., 2019).

Regulation of AC by C-Raf plays a key role in models of superactivation of AC precipitated by removal of agonist after sustained treatment with morphine or agonists of other Gicoupled receptors. Inhibition of C-Raf attenuates superactivation of AC that occurs after long-term opioid exposure (Varga et al., 2002). Additionally, inhibition or knockdown of C-Raf blunts the development of thermal hyperalgesia, mechanical allodynia, and antinociceptive tolerance induced by sustained morphine treatment (Tumati et al., 2008). Although superactivation, like SCI and depolarization, involves C-Raf, the similarities end there. Unlike superactivation, neither depolarization nor SCI enhanced Fsk-stimulated cAMP production. Additionally, depolarizationmediated reduction in DAMGO sensitivity occurred within $5 \mathrm{~min}$, rather than the $2-12 \mathrm{~h}$ required for sustained morphineinduced superactivation (Varga et al., 2002). Moreover, superactivation models depend on Src activation of C-Raf (Zhang et al., 2013), whereas we found no effect of Src inhibition on depolarization-induced reductions of DAMGO potency. These differences suggest that the mechanism by which superactivation versus $\mathrm{SCI}$ or depolarization engages $\mathrm{C}$-Raf for regulation of AC is distinct and context-dependent.

\section{Functional implications of depolarization-dependent C-Raf-} ERK and C-Raf-AC signaling in nociceptors

In many experimental models of persistent pain, a continuing depolarization of nociceptor RMP remains after excision of the ganglion or dissociation of the neurons. These include nociceptors investigated not only in rodent SCI models (Odem et al., 2018; Berkey et al., 2020), but also in peripheral axotomy models in rats (e.g., Sapunar et al., 2005) and Aplysia (Ungless et al., 2002), and other rodent pain models, such as peripheral inflammation (Qu et al., 2014), chemotherapy (Y. Li et al., 2017), bone cancer (Zheng et al., 2012), and chronic compression of the DRG (Song et al., 2006). Nociceptor hyperexcitability manifested as enhanced repetitive firing (including SA), reduced AP threshold, 
and/or depolarized RMP has been linked to cAMP signaling through PKA, EPAC, or HCN channels in several of these models (Aley and Levine, 1999; Bavencoffe et al., 2016; Djouhri et al., 2018; Berkey et al., 2020; for review, see Z. H. Li et al., 2019). In addition, ERK signaling enhances nociceptor function in various pain-related models (e.g., Obata et al., 2003; Ji et al., 2009; Ferrari et al., 2014; Y. Li et al., 2015; Mihail et al., 2019). Little is known about how ERK increases nociceptor excitability, although evidence suggests that ERK can directly phosphorylate sodium channel Nav1.7, allowing it to open at more hyperpolarized membrane potentials and perhaps contribute to a more depolarized RMP (Stamboulian et al., 2010). The present study shows that substantial enhancement of the basal activity of AC and ERK activity is produced by modest depolarization of RMP into the RMP range that occurs naturally in hyperactive nociceptors during persistent pain states. This discovery has important implications. First, it indicates that depolarization of RMP in these states increases the $\mathrm{OA}$ of nociceptors not only by bringing membrane potential closer to AP threshold, but also by increasing the activity of major cell signaling pathways that themselves promote hyperexcitability. Second, the positive feedback between the basal activity of AC and ERK signaling pathways on the one hand and RMP on the other should by itself contribute to the persistence of pain driven by $\mathrm{OA}$ in nociceptors. This positive feedback loop may reinforce persistent hyperexcitable effects mediated by the transcriptional and translational consequences of signaling by AC and ERK (e.g., Obata et al., 2003; Ferrari et al., 2015). Moreover, the C-Raf-mediated reduction in sensitivity of $\mathrm{AC}$ to $\mathrm{G} \alpha \mathrm{i}$ in depolarized nociceptors should further enhance nociceptor activity by reducing suppressive effects of endogenous opioids. Thus, depolarization-dependent C-Raf-ERK and C-RafAC signaling should promote nociceptor hyperactivity by multiple, self-reinforcing mechanisms, some of which may reduce the efficacy of clinically applied opioids and cannabinoids. These findings raise important questions about how C-Raf-dependent, MOR-sensitive, nociceptor hyperactivity may contribute to spontaneous pain (Yang et al., 2014), as well as to evoked pain (Sun et al., 2019) and MOR-dependent pathophysiology, such as hyperalgesia, Type II hyperalgesic priming, and opioid tolerance (Araldi et al., 2017, 2019; Corder et al., 2017).

\section{References}

Aley K, Levine J (1999) Role of protein kinase A in the maintenance of inflammatory pain. J Neurosci 19:2181-2186.

Araldi D, Ferrari LF, Levine JD (2017) Hyperalgesic priming (type II) induced by repeated opioid exposure: maintenance mechanisms. Pain 158:1204-1216.

Araldi D, Bogen O, Green PG, Levine JD (2019) Role of nociceptor Toll-like receptor 4 (TLR4) in opioid-induced hyperalgesia and hyperalgesic priming. J Neurosci 39:6414-6424.

Baldassa S, Zippel R, Sturani E (2003) Depolarization-induced signaling to Ras, Rap1 and MAPKs in cortical neurons. Brain Res Mol Brain Res 119:111-122.

Barry PH (1994) JPCalc, a software package for calculating liquid junction potential corrections in patch-clamp, intracellular, epithelial and bilayer measurements and for correcting junction potential measurements. J Neurosci Methods 51:107-116.

Basso DM, Beattie MS, Bresnahan JC (1995) A sensitive and reliable locomotor rating scale for open field testing in rats. J Neurotrauma 12:1-21.

Bavencoffe A, Li Y, Wu Z, Yang Q, Herrera J, Kennedy EJ, Walters ET, Dessauer CW (2016) Persistent electrical activity in primary nociceptors after spinal cord injury is maintained by scaffolded adenylyl cyclase and protein kinase $\mathrm{A}$ and is associated with altered adenylyl cyclase regulation. J Neurosci 36:1660-1668.
Beazely MA, Alan JK, Watts VJ (2005) Protein kinase C and epidermal growth factor stimulation of Raf1 potentiates adenylyl cyclase type 6 activation in intact cells. Mol Pharmacol 67:250-259.

Bedi S, Yang Q, Crook R, Du J, Wu Z, Fishman H, Grill R, Carlton S, Walters E (2010) Chronic spontaneous activity generated in the somata of primary nociceptors is associated with pain-related behavior after spinal cord injury. J Neurosci 30:14870-14882.

Berkey SC, Herrera JJ, Odem MA, Rahman S, Cheruvu SS, Cheng X, Walters ET, Dessauer CW, Bavencoffe AG (2020) EPAC1 and EPAC2 promote nociceptor hyperactivity associated with chronic pain after spinal cord injury. Neurobiol Pain 7:100040.

Bryce TN (2018) Opioids should not be prescribed for chronic pain after spinal cord injury. Spinal Cord Ser Cases 4:66.

Carlton S, Du J, Tan H, Nesic O, Hargett G, Bopp A, Yamani A, Lin Q, Willis W, Hulsebosch C (2009) Peripheral and central sensitization in remote spinal cord regions contribute to central neuropathic pain after spinal cord injury. Pain 147:265-276.

Corbit KC, Trakul N, Eves EM, Diaz B, Marshall M, Rosner MR (2003) Activation of Raf- 1 signaling by protein kinase $\mathrm{C}$ through a mechanism involving Raf kinase inhibitory protein. J Biol Chem 278:13061-13068.

Corder G, Tawfik VL, Wang D, Sypek EI, Low SA, Dickinson JR, Sotoudeh C, Clark JD, Barres BA, Bohlen CJ, Scherrer G (2017) Loss of mu opioid receptor signaling in nociceptors, but not microglia, abrogates morphine tolerance without disrupting analgesia. Nat Med 23:164-173.

de Hoon MJ, Imoto S, Nolan J, Miyano S (2004) Open source clustering software. Bioinformatics 20:1453-1454.

Dessauer CW, Watts VJ, Ostrom RS, Conti M, Dove S, Seifert R (2017) International Union of Basic and Clinical Pharmacology: CI. Structures and small molecule modulators of mammalian adenylyl cyclases. Pharmacol Rev 69:93-139.

Ding Q, Gros R, Gray ID, Taussig R, Ferguson SS, Feldman RD (2004) Raf kinase activation of adenylyl cyclases: isoform-selective regulation. Mol Pharmacol 66:921-928.

Djouhri L, Smith T, Ahmeda A, Alotaibi M, Weng X (2018) Hyperpolarization-activated cyclic nucleotide-gated channels contribute to spontaneous activity in L4 C-fiber nociceptors, but not Abeta-nonnociceptors, after axotomy of L5-spinal nerve in the rat in vivo. Pain 159:1392-1402.

Du X, Hao H, Gigout S, Huang D, Yang Y, Li L, Wang C, Sundt D, Jaffe DB, Zhang H, Gamper N (2014) Control of somatic membrane potential in nociceptive neurons and its implications for peripheral nociceptive transmission. Pain 155:2306-2322.

Ferrari LF, Bogen O, Levine JD (2014) Second messengers mediating the expression of neuroplasticity in a model of chronic pain in the rat. J Pain 15:312-320.

Ferrari LF, Araldi D, Levine JD (2015) Distinct terminal and cell body mechanisms in the nociceptor mediate hyperalgesic priming. J Neurosci 35:6107-6116.

Inder K, Harding A, Plowman SJ, Philips MR, Parton RG, Hancock JF (2008) Activation of the MAPK module from different spatial locations generates distinct system outputs. Mol Biol Cell 19:4776-4784.

Isensee J, Diskar M, Waldherr S, Buschow R, Hasenauer J, Prinz A, Allgower F, Herberg FW, Hucho T (2014) Pain modulators regulate the dynamics of PKA-RII phosphorylation in subgroups of sensory neurons. J Cell Sci 127:216-229.

Isensee J, Krahe L, Moeller K, Pereira V, Sexton JE, Sun X, Emery E, Wood JN, Hucho T (2017) Synergistic regulation of serotonin and opioid signaling contributes to pain insensitivity in Nav1.7 knockout mice. Sci Signal 10:eaah4874.

Isensee J, Kaufholz M, Knape MJ, Hasenauer J, Hammerich $\mathrm{H}$, Gonczarowska-Jorge H, Zahedi RP, Schwede F, Herberg FW, Hucho T (2018) PKA-RII subunit phosphorylation precedes activation by cAMP and regulates activity termination. J Cell Biol 217:2167-2184.

Ji RR, Gereau RW, Malcangio M, Strichartz GR (2009) MAP kinase and pain. Brain Res Rev 60:135-148.

Kramer JL, Minhas NK, Jutzeler CR, Erskine EL, Liu LJ, Ramer MS (2017) Neuropathic pain following traumatic spinal cord injury: models, measurement, and mechanisms. J Neurosci Res 95:1295-1306.

Leicht DT, Balan V, Kaplun A, Singh-Gupta V, Kaplun L, Dobson M, Tzivion G (2007) Raf kinases: function, regulation and role in human cancer. Biochim Biophys Acta 1773:1196-1212. 
Li Y, Zhang H, Kosturakis AK, Cassidy RM, Zhang H, Kennamer-Chapman RM, Jawad AB, Colomand CM, Harrison DS, Dougherty PM (2015) MAPK signaling downstream to TLR4 contributes to paclitaxel-induced peripheral neuropathy. Brain Behav Immun 49:255-266.

Li Y, Tatsui CE, Rhines LD, North RY, Harrison DS, Cassidy RM, Johansson CA, Kosturakis AK, Edwards DD, Zhang H, Dougherty PM (2017) Dorsal root ganglion neurons become hyperexcitable and increase expression of voltage-gated T-type calcium channels (Cav3.2) in paclitaxel-induced peripheral neuropathy. Pain 158:417-429.

Li ZH, Cui D, Qiu CJ, Song XJ (2019) Cyclic nucleotide signaling in sensory neuron hyperexcitability and chronic pain after nerve injury. Neurobiol Pain 6:100028.

Liu S, Huang Q, He S, Chen Z, Gao X, Ma D, Duan W, Ford N, Yang F, Chen X, Raja SN, Hao D, Guan Y (2019) Dermorphin [D-Arg2, Lys4] (14) amide inhibits below-level heat hypersensitivity in mice after contusive thoracic spinal cord injury. Pain 160:2710-2723.

McLaughlin KA, Levin M (2018) Bioelectric signaling in regeneration: mechanisms of ionic controls of growth and form. Dev Biol 433:177-189.

Mihail SM, Wangzhou A, Kunjilwar KK, Moy JK, Dussor G, Walters ET, Price TJ (2019) MNK-eIF4E signalling is a highly conserved mechanism for sensory neuron axonal plasticity: evidence from Aplysia californica. Philos Trans R Soc Lond B Biol Sci 374:20190289.

Mogil JS (2020) Qualitative sex differences in pain processing: emerging evidence of a biased literature. Nat Rev Neurosci 21:353-365.

Obata K, Yamanaka H, Dai Y, Tachibana T, Fukuoka T, Tokunaga A, Yoshikawa H, Noguchi K (2003) Differential activation of extracellular signal-regulated protein kinase in primary afferent neurons regulates brain-derived neurotrophic factor expression after peripheral inflammation and nerve injury. J Neurosci 23:4117-4126.

Odem MA, Bavencoffe AG, Cassidy RM, Lopez ER, Tian J, Dessauer CW, Walters ET (2018) Isolated nociceptors reveal multiple specializations for generating irregular ongoing activity associated with ongoing pain. Pain 159:2347-2362.

Polakiewicz RD, Schieferl SM, Dorner LF, Kansra V, Comb MJ (1998) A mitogen-activated protein kinase pathway is required for mu-opioid receptor desensitization. J Biol Chem 273:12402-12406.

Qu L, Fan N, Ma C, Wang T, Han L, Fu K, Wang Y, Shimada SG, Dong X, LaMotte RH (2014) Enhanced excitability of MRGPRA3- and MRGPRD-positive nociceptors in a model of inflammatory itch and pain. Brain 137:1039-1050.

Ritter DM, Zemel BM, Hala TJ, O'Leary ME, Lepore AC, Covarrubias M (2015) Dysregulation of Kv3.4 channels in dorsal root ganglia following spinal cord injury. J Neurosci 35:1260-1273.

Rodríguez-Muñoz M, de la Torre-Madrid E, Sánchez-Blázquez P, Garzón J (2011) NO-released zinc supports the simultaneous binding of Raf- 1 and PKCgamma cysteine-rich domains to HINT1 protein at the mu-opioid receptor. Antioxid Redox Signal 14:2413-2425.

Roederer M (2002) Compensation in flow cytometry. Curr Protoc Cytom Chapter 1:Unit 114.

Rosen LB, Ginty DD, Weber MJ, Greenberg ME (1994) Membrane depolarization and calcium influx stimulate MEK and MAP kinase via activation of Ras. Neuron 12:1207-1221.

Ruland JG, Kirchhofer SB, Klindert S, Bailey CP, Bunemann M (2020) Voltage modulates the effect of mu-receptor activation in a ligand-dependent manner. Br J Pharmacol 177:3489-3504.

Salzer I, Ray S, Schicker K, Boehm S (2019) Nociceptor signalling through ion channel regulation via GPCRs. Int J Mol Sci 20:2488.

Sapunar D, Modric-Jednacak K, Grkovic I, Michalkiewicz M, Hogan QH (2005) Effect of peripheral axotomy on pain-related behavior and dorsal root ganglion neurons excitability in NPY transgenic rats. Brain Res 1063:48-58

Song X, Wang Z, Gan Q, Walters E (2006) cAMP and cGMP contribute to sensory neuron hyperexcitability and hyperalgesia in rats with dorsal root ganglia compression. J Neurophysiol 95:479-492.

Stamboulian S, Choi JS, Ahn HS, Chang YW, Tyrrell L, Black JA, Waxman SG, Dib-Hajj SD (2010) ERK1/2 mitogen-activated protein kinase phosphorylates sodium channel $\mathrm{Na}(\mathrm{v}) 1.7$ and alters its gating properties. J Neurosci 30:1637-1647.

Strassheim D, Malbon CC (1994) Phosphorylation of Gi alpha 2 attenuates inhibitory adenylyl cyclase in neuroblastoma/glioma hybrid (NG-108-15) cells. J Biol Chem 269:14307-14313.

Sun J, Chen SR, Chen H, Pan HL (2019) mu-Opioid receptors in primary sensory neurons are essential for opioid analgesic effect on acute and inflammatory pain and opioid-induced hyperalgesia. J Physiol 597:16611675.

Tan CM, Kelvin DJ, Litchfield DW, Ferguson SS, Feldman RD (2001) Tyrosine kinase-mediated serine phosphorylation of adenylyl cyclase. Biochemistry 40:1702-1709.

Tumati S, Milnes TL, Yamamura HI, Vanderah TW, Roeske WR, Varga EV (2008) Intrathecal Raf-1-selective siRNA attenuates sustained morphinemediated thermal hyperalgesia. Eur J Pharmacol 601:207-208.

Ungless MA, Gasull X, Walters ET (2002) Long-term alteration of S-type potassium current and passive membrane properties in Aplysia sensory neurons following axotomy. J Neurophysiol 87:2408-2420.

Varga EV, Rubenzik M, Grife V, Sugiyama M, Stropova D, Roeske WR, Yamamura HI (2002) Involvement of Raf-1 in chronic delta-opioid receptor agonist-mediated adenylyl cyclase superactivation. Eur J Pharmacol 451:101-102.

Vickery ON, Machtens JP, Zachariae U (2016) Membrane potentials regulating GPCRs: insights from experiments and molecular dynamics simulations. Curr Opin Pharmacol 30:44-50.

Vierck C (2020) Mechanisms of below-level pain following spinal cord injury (SCI). J Pain 21:262-280.

Wang HB, Zhao B, Zhong YQ, Li KC, Li ZY, Wang Q, Lu YJ, Zhang ZN, He SQ, Zheng HC, Wu SX, Hokfelt TG, Bao L, Zhang X (2010) Coexpression of delta- and mu-opioid receptors in nociceptive sensory neurons. Proc Natl Acad Sci USA 107:13117-13122.

Widerstrom-Noga E (2017) Neuropathic pain and spinal cord injury: phenotypes and pharmacological management. Drugs 77:967-984.

Wu Z, Yang Q, Crook RJ, O’Neil RG, Walters ET (2013) TRPV1 channels make major contributions to behavioral hypersensitivity and spontaneous activity in nociceptors after spinal cord injury. Pain 154:2130-2141.

Wu Z, Li L, Xie F, Du J, Zuo Y, Frost JA, Carlton SM, Walters ET, Yang Q (2017) Activation of KCNQ channels suppresses spontaneous activity in dorsal root ganglion neurons and reduces chronic pain after spinal cord injury. J Neurotrauma 34:1260-1270.

Yang Q, Wu Z, Hadden JK, Odem MA, Zuo Y, Crook RJ, Frost JA, Walters ET (2014) Persistent pain after spinal cord injury is maintained by primary afferent activity. J Neurosci 34:10765-10769.

Zhang L, Loh HH, Law PY (2013) A novel noncanonical signaling pathway for the mu-opioid receptor. Mol Pharmacol 84:844-853.

Zhang X, Bao L, Li S (2015) Opioid receptor trafficking and interaction in nociceptors. Br J Pharmacol 172:364-374.

Zheng Q, Fang D, Cai J, Wan Y, Han JS, Xing GG (2012) Enhanced excitability of small dorsal root ganglion neurons in rats with bone cancer pain. Mol Pain 8:24. 